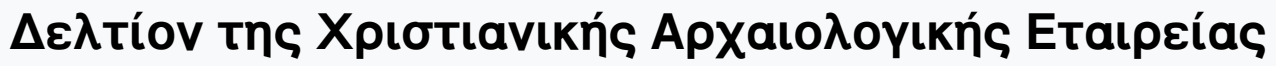

Tóp. 6, Ap. 1 (1906)

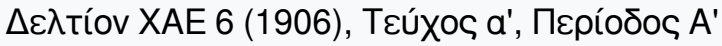

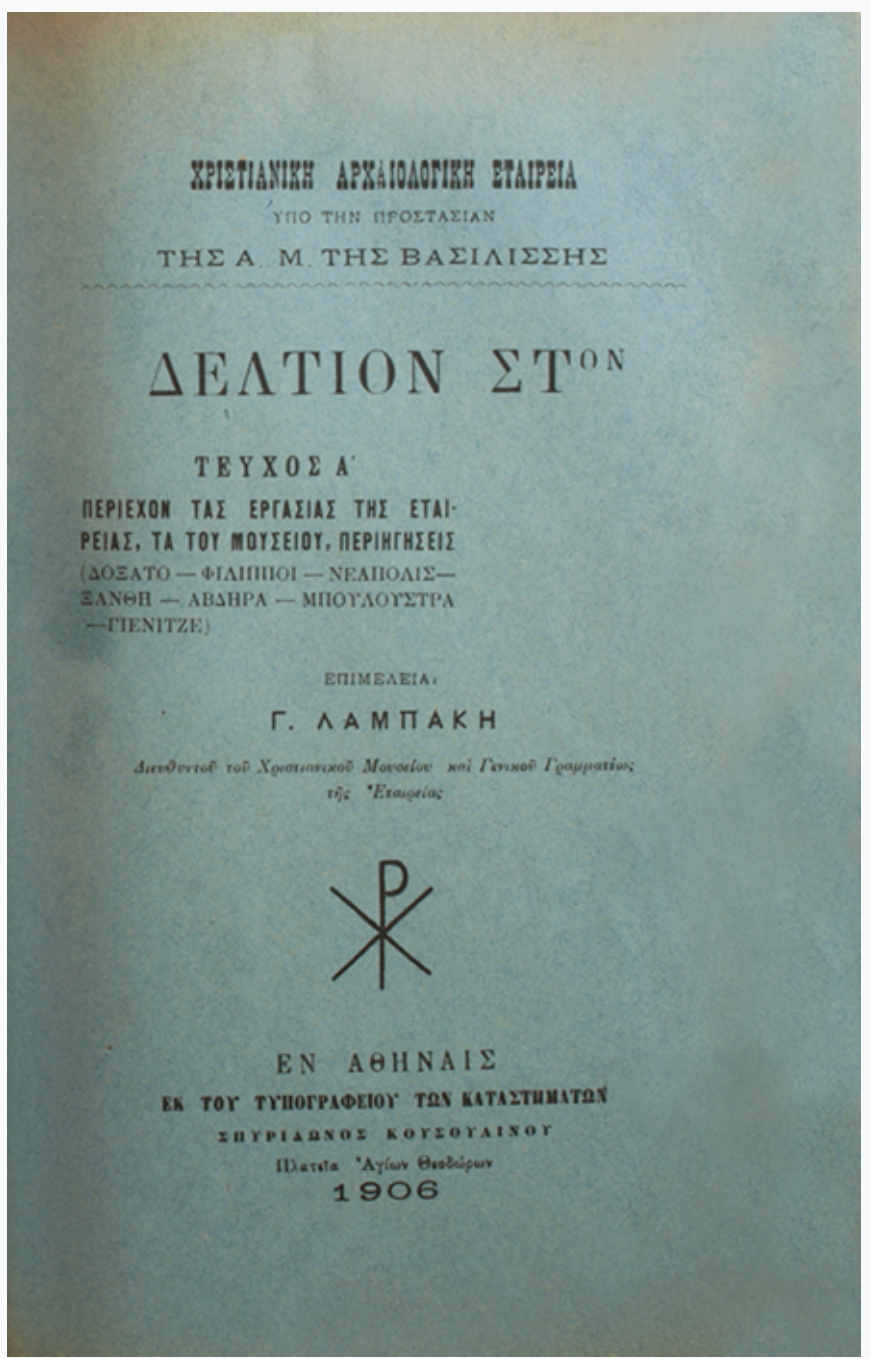

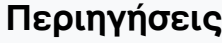

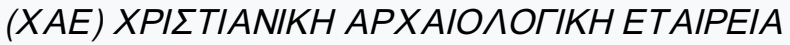

doi: $10.12681 /$ dchae.1612

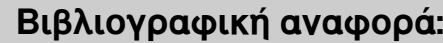

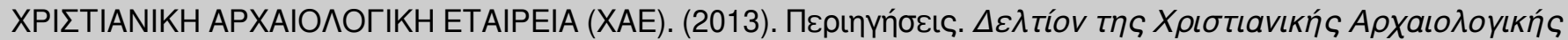
Eтaıрía s, 6(1), 22-48. https://doi.org/10.12681/dchae.1612 


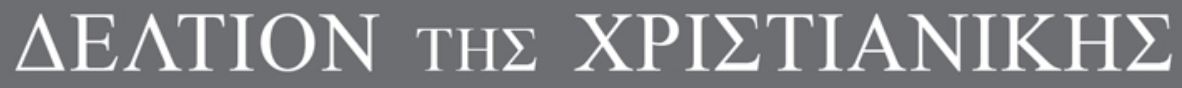 APXAIO $\Lambda$ OГIKH $\Sigma$ ETAIPEIA $\Sigma$}

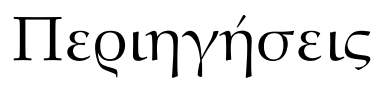

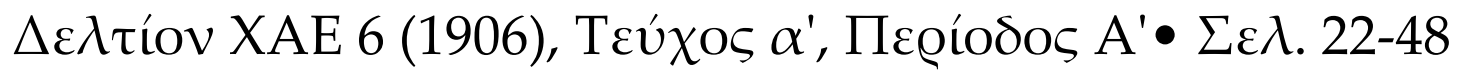
A@HNA 1906 


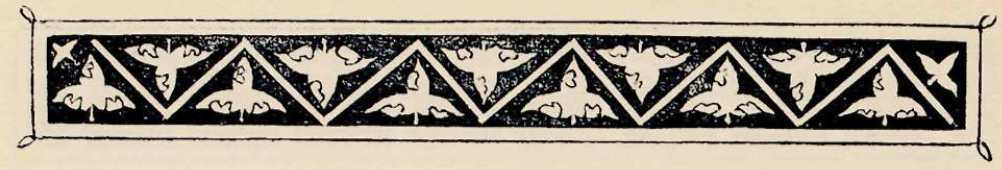

\section{ПЕРIHГН ЕI}

\section{$\triangle O \equiv A T O$}

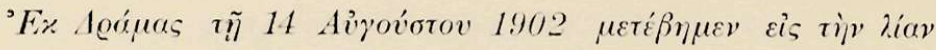

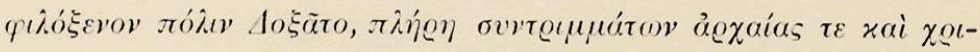

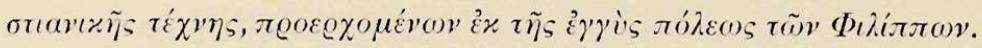

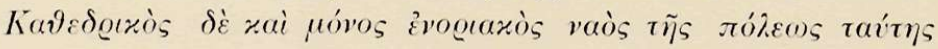

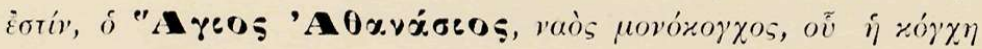
xงหนหนi.

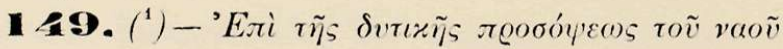

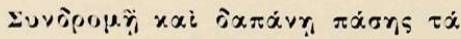

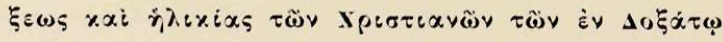

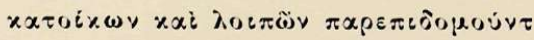

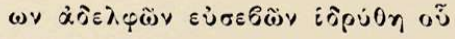

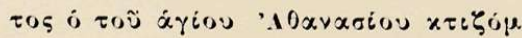

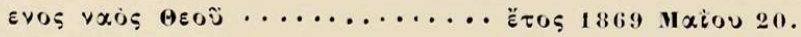

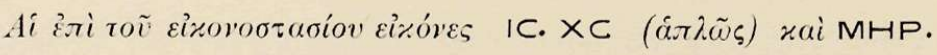

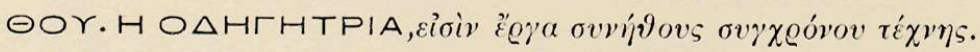

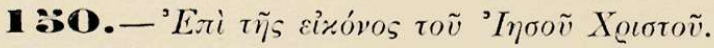

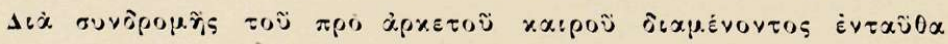

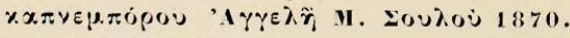

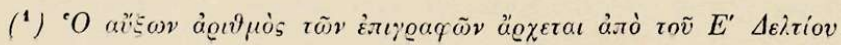




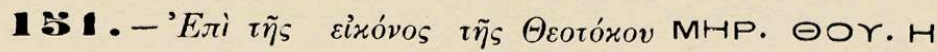
ODHTHTPIA

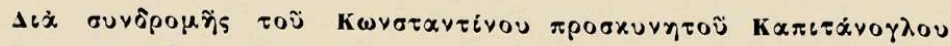

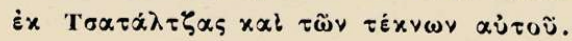

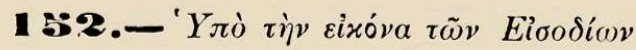

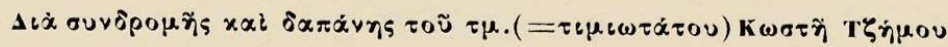

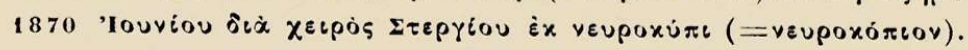

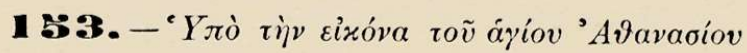

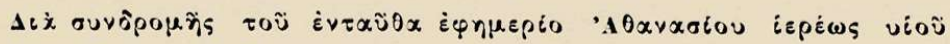

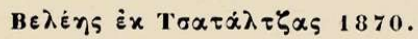

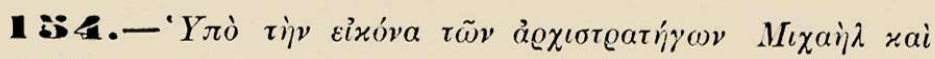

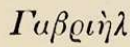

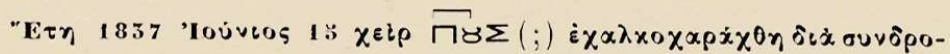

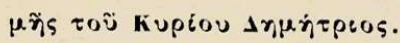

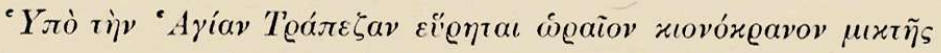

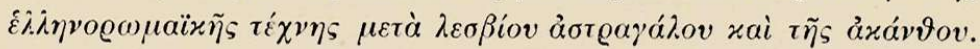

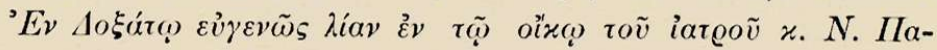

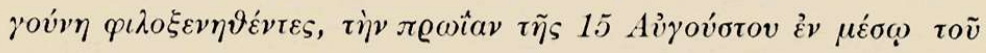

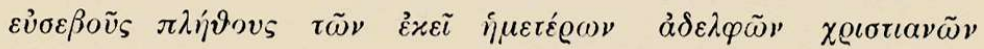

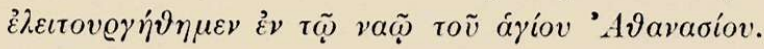

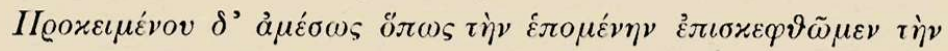

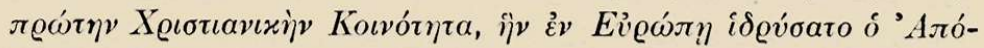

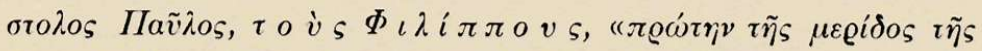

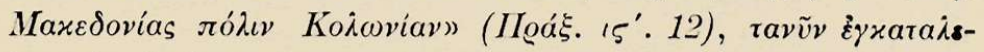

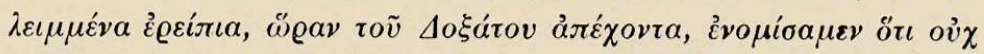

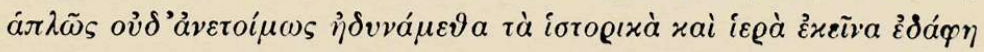

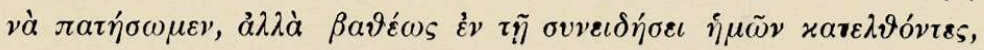

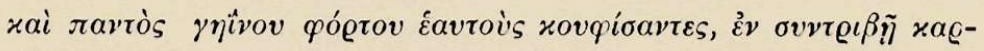

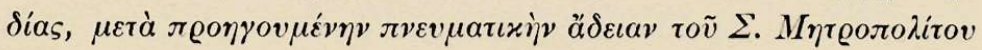

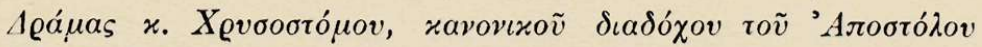

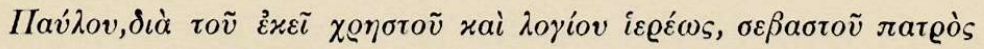

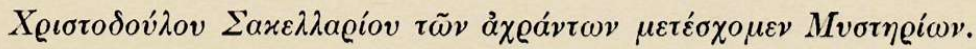


Ö̈т(1) $\delta$ '

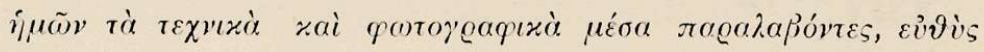

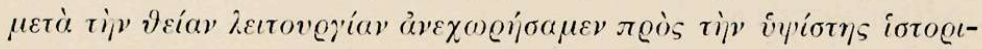

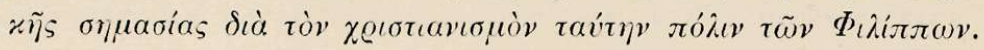

\section{Ф। $\wedge \Pi \Pi$ । ।}

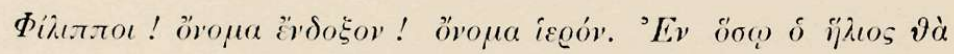

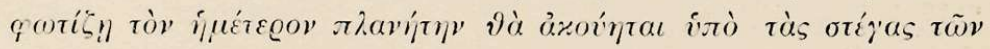

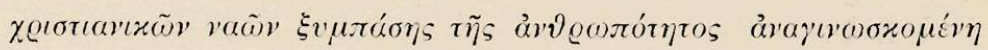

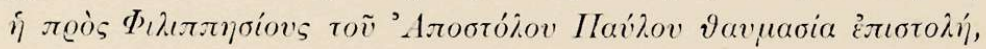

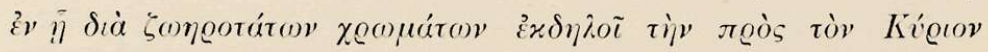

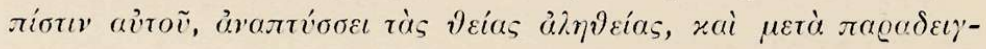

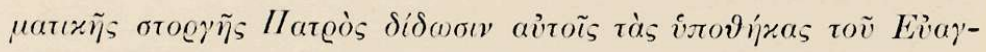

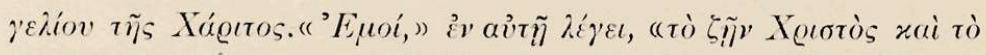

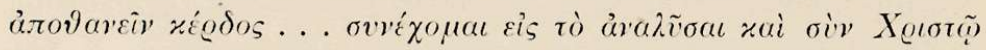

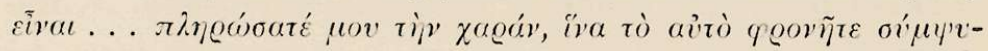

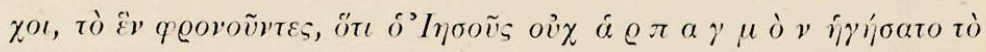

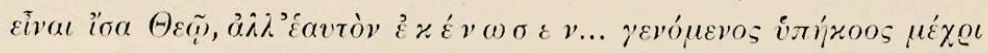

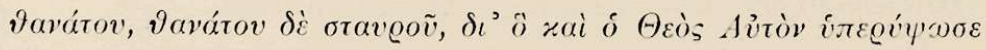

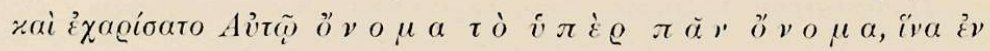

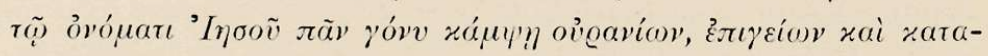

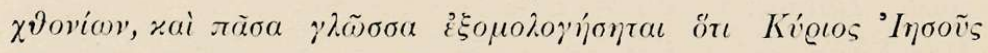

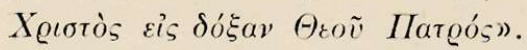

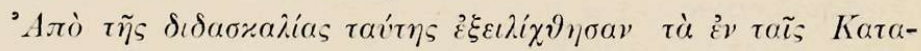

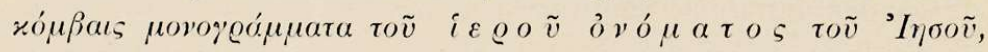

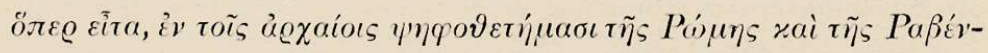

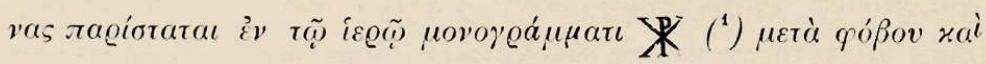

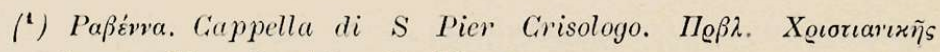

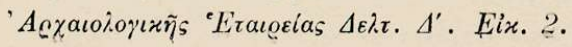




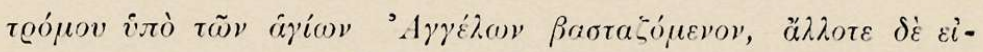

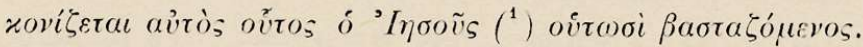

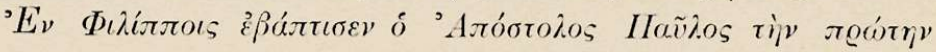

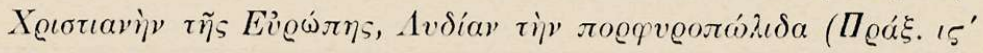

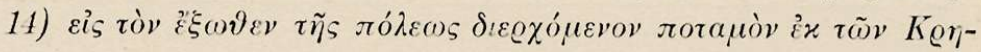

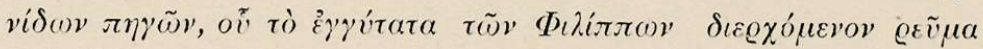

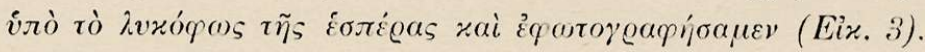

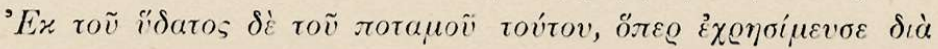

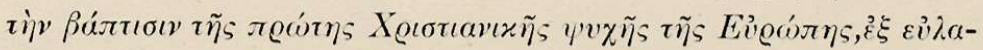

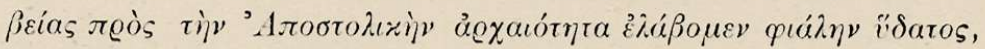

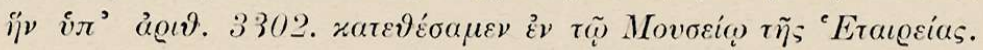

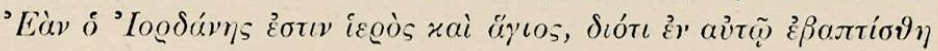

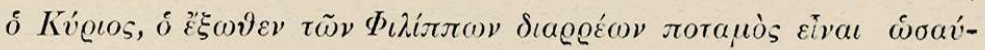

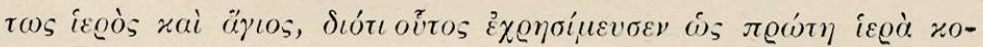

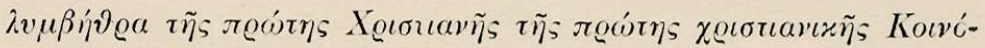

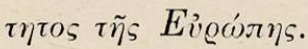

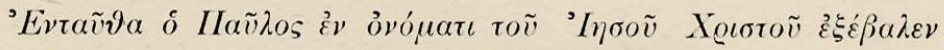

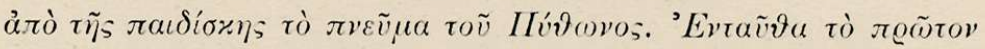

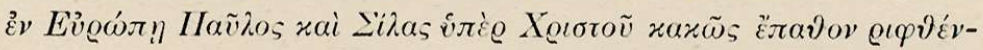

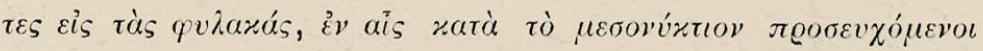

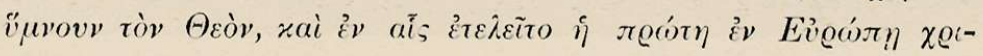

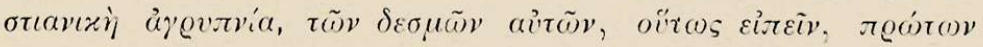

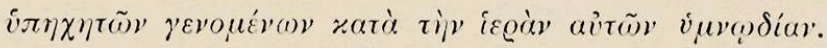

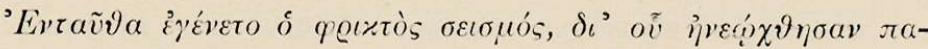

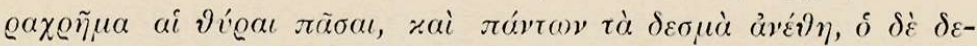

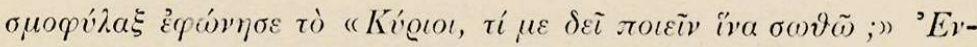

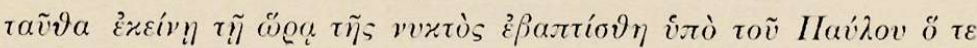

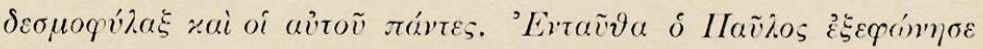

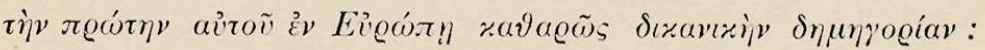

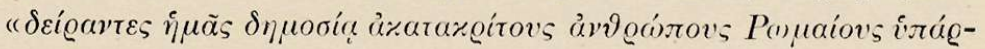

(1) 'Ev Póm. Cappella S. Zenone żv $\tau \tilde{\eta}$ S. Prussede. Anton de

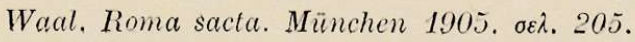




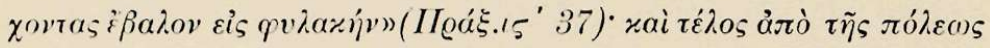

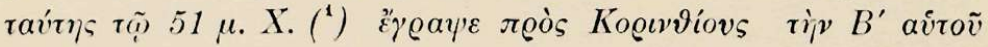

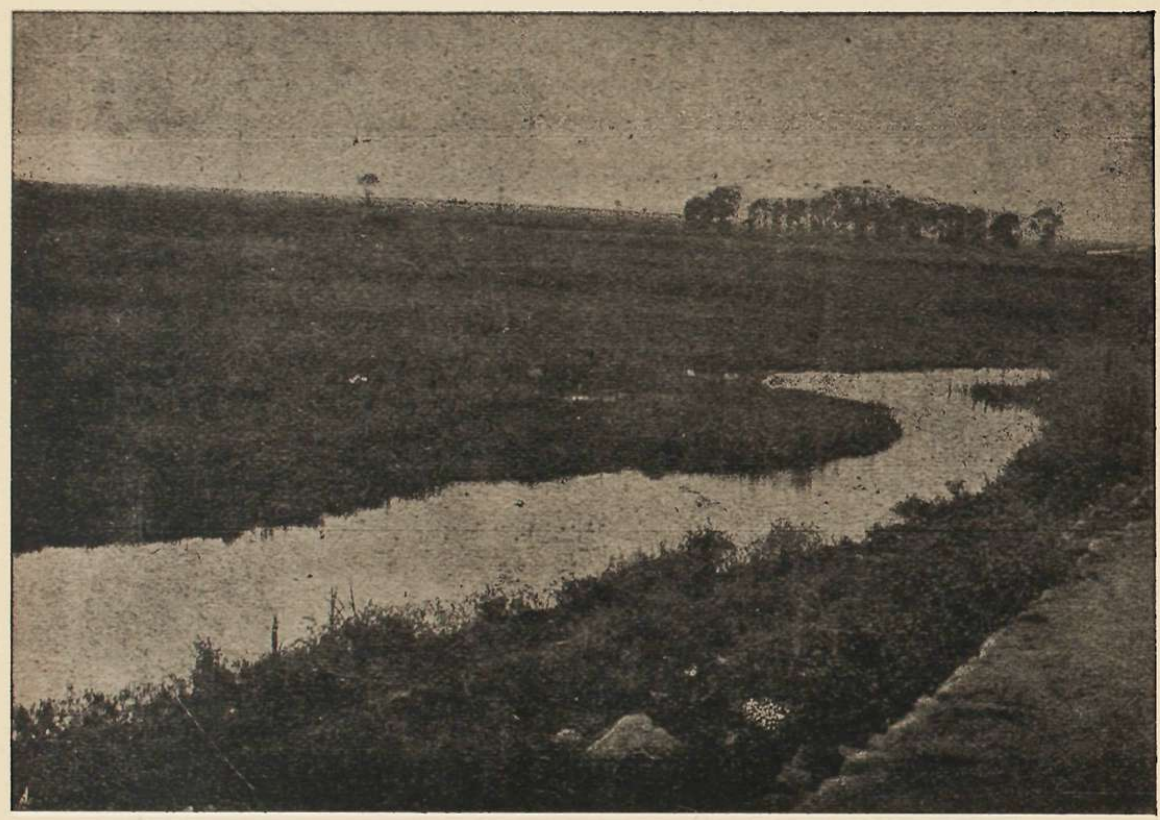

E'xisy 3.

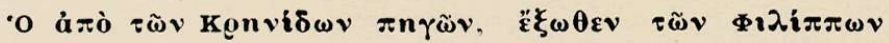

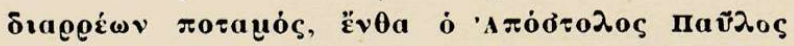

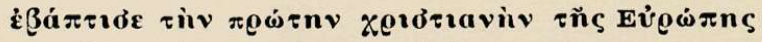

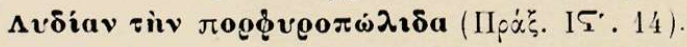

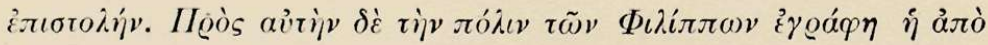

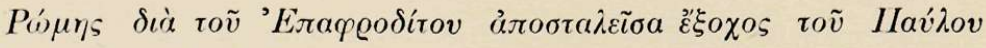

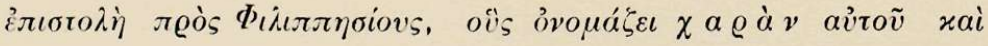

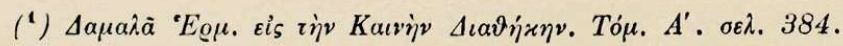




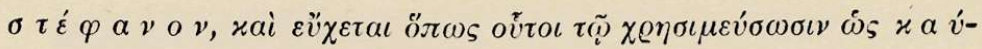
$\chi \eta \mu \alpha \quad \varkappa \alpha \tau \dot{\alpha} \quad \tau \dot{\eta} \nu \quad \dot{\eta} \mu \dot{\varepsilon} \varrho \alpha \nu \quad \dot{\varepsilon} \varkappa \varepsilon i v \eta \nu \quad \tau \tilde{\eta} \varsigma \quad \vec{\varepsilon} \pi \iota \varphi \alpha-$

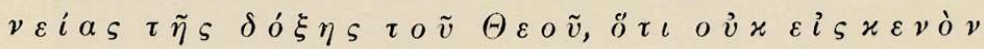

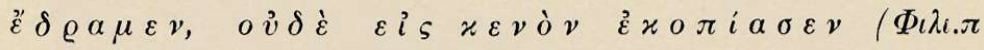
$\left.\beta^{\prime} 16\right)$.

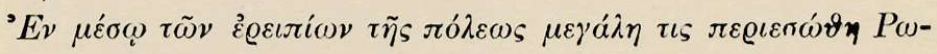

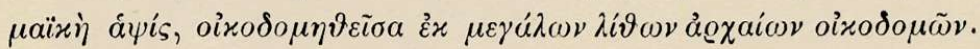
(Eix. 4).

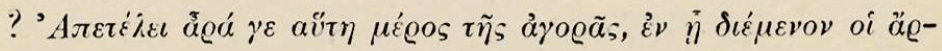

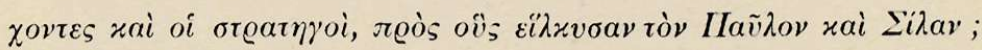

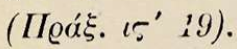

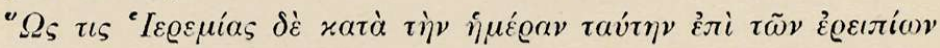

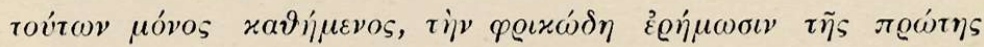

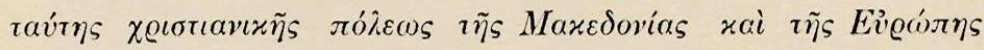

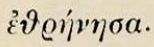

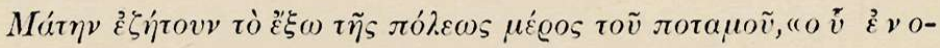

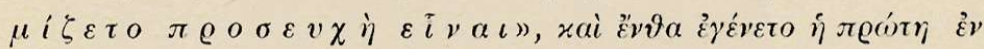

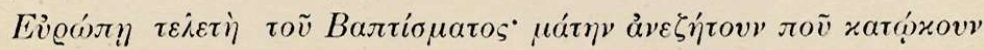

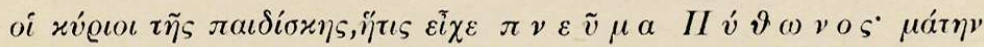

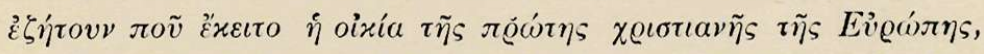

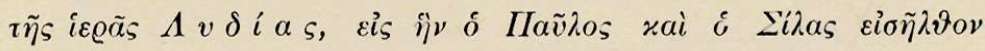

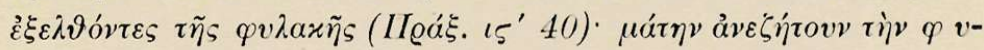

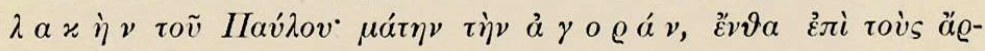

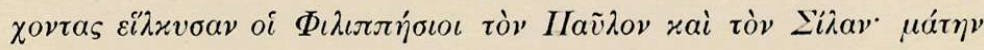

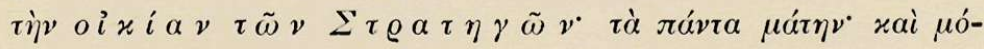

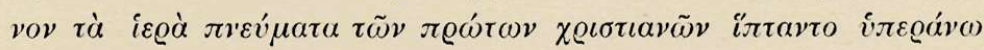

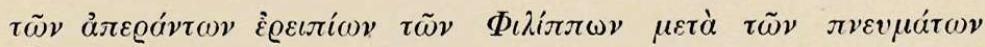

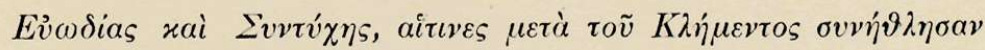

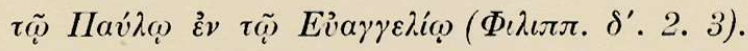

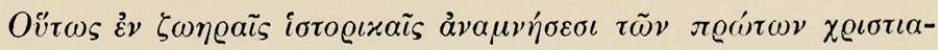

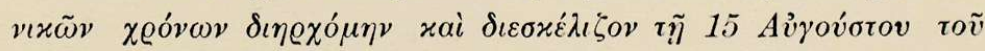

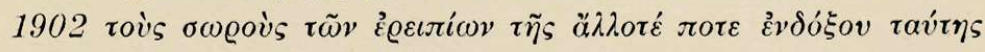

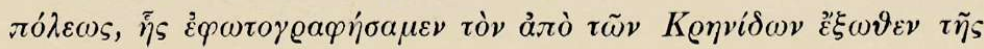




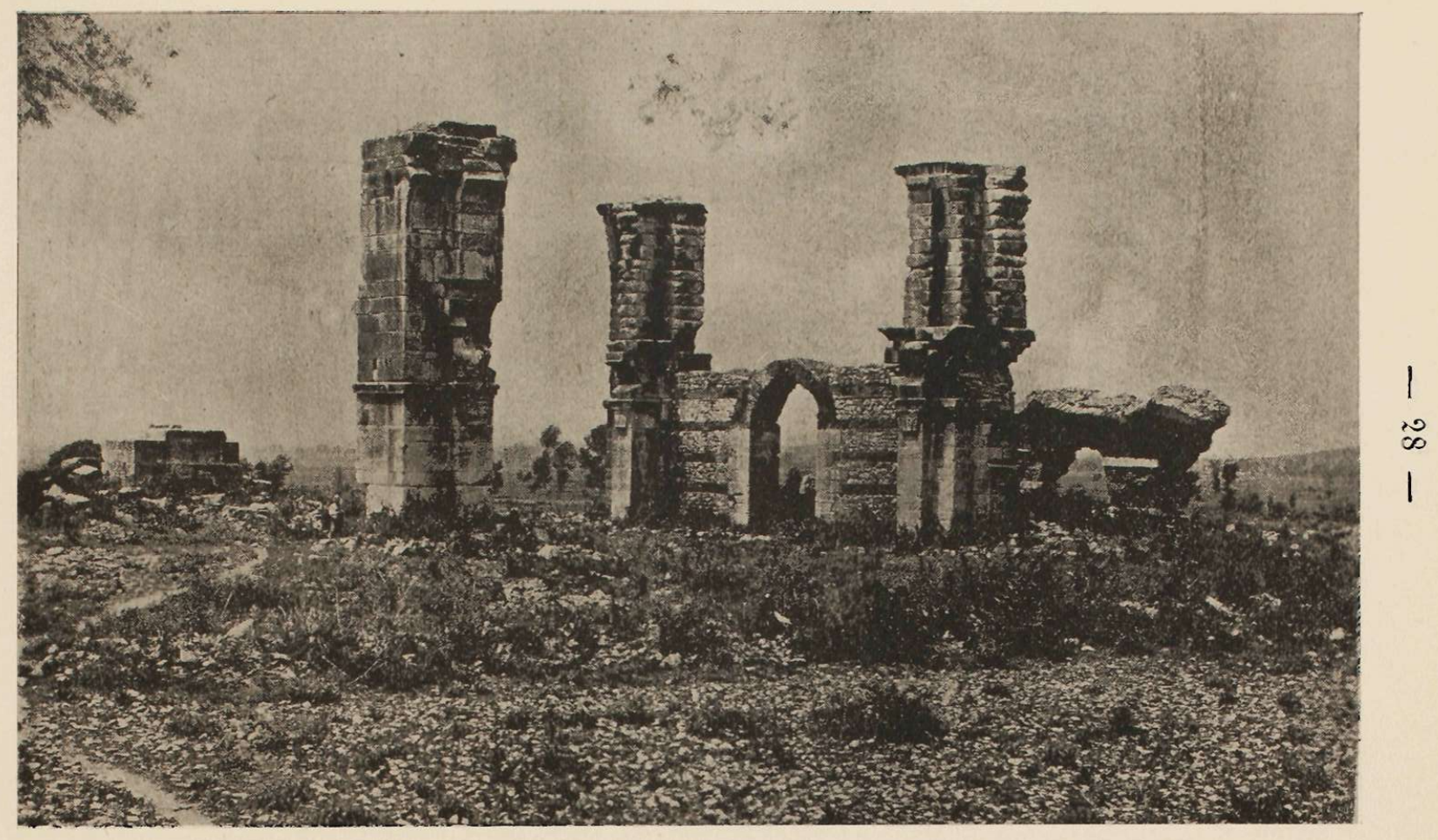

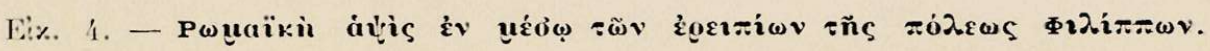




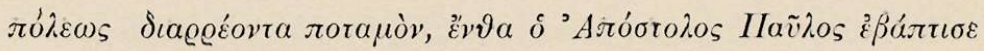

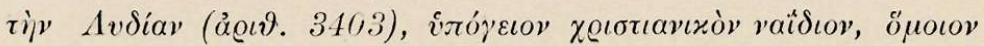

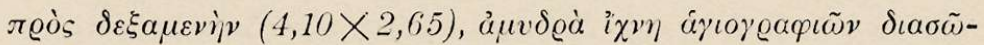

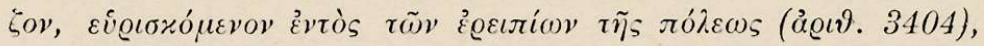

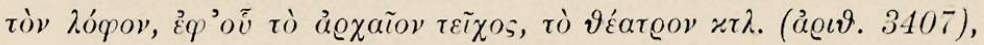

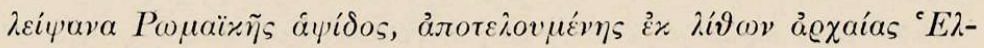

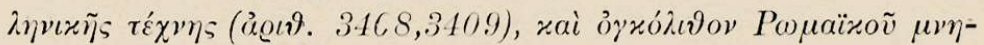

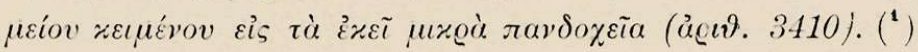

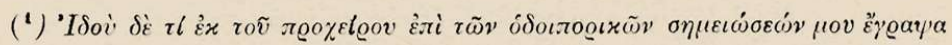

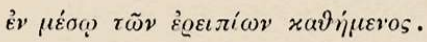

\section{$\Phi i \lambda, \pi \pi 0$ i.}

\section{5. Á̉yoúozov 1902.}

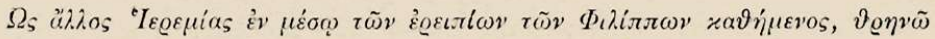

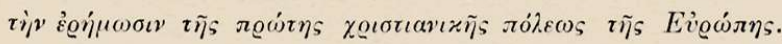

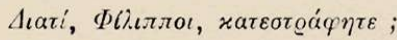

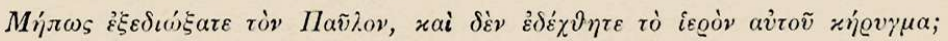

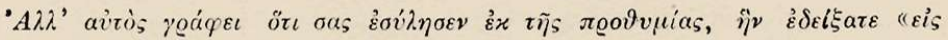

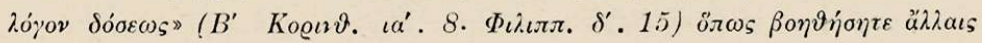

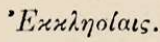

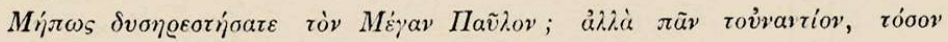

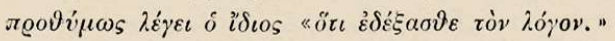

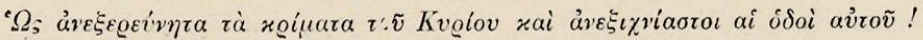

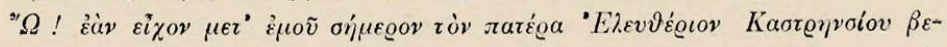

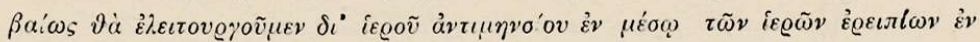

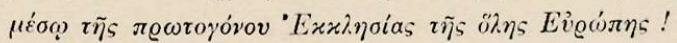

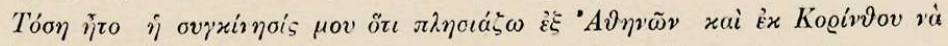

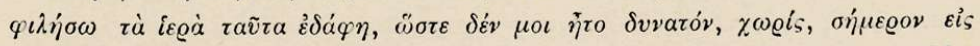

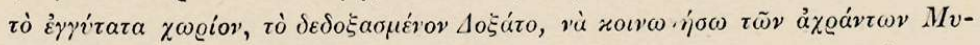

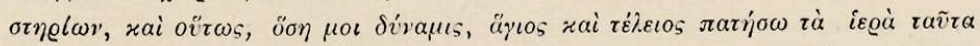

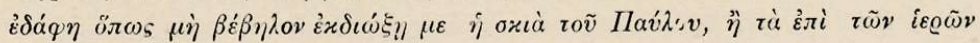

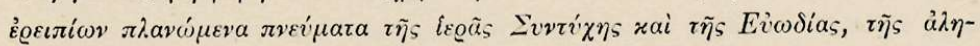

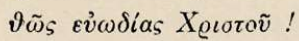

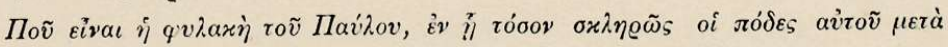

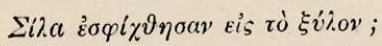

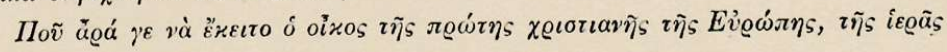




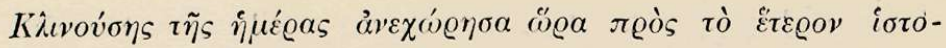

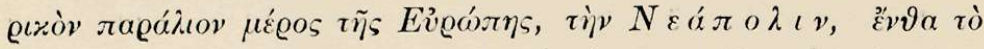

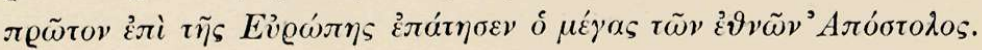

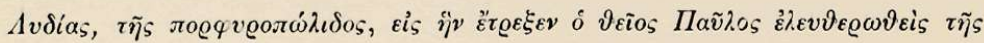
$q v \lambda \alpha x \tilde{\eta} \varsigma$;

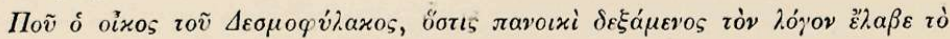

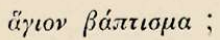

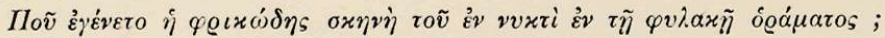

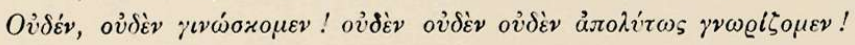

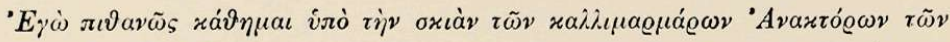

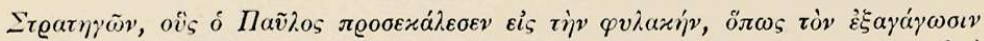

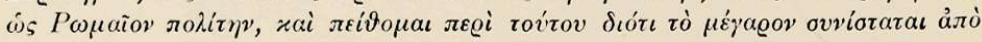

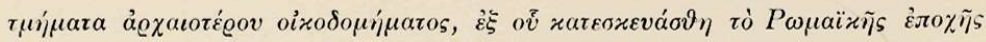

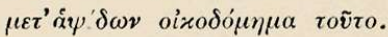

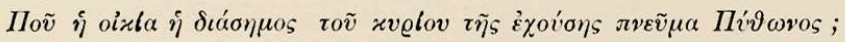

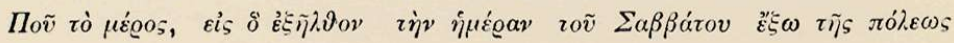

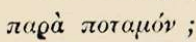

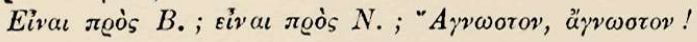

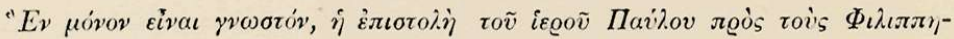

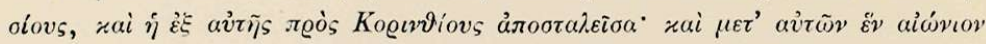

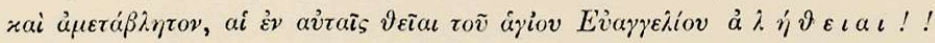

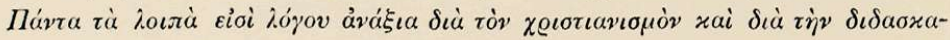

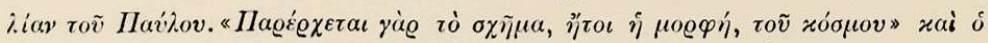

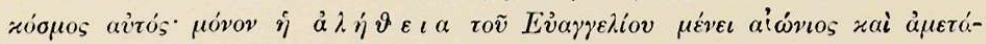

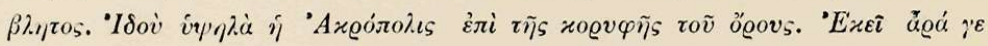

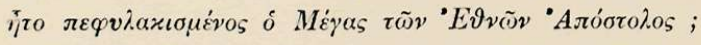

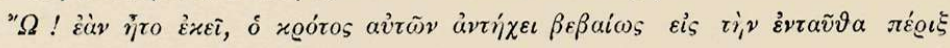

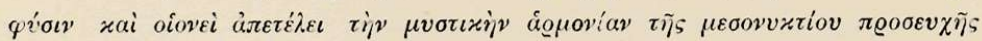

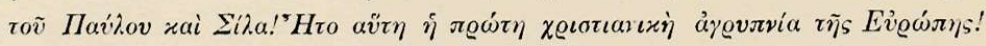

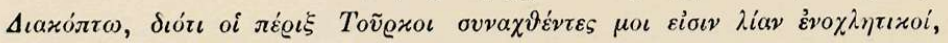

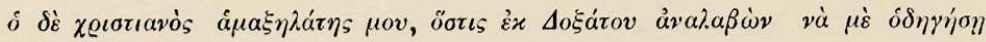

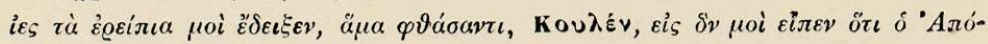

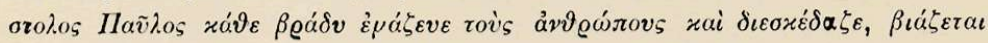

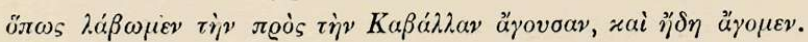

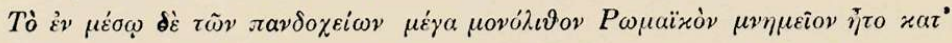

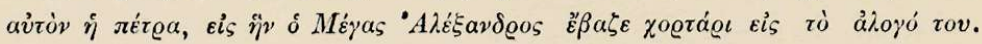

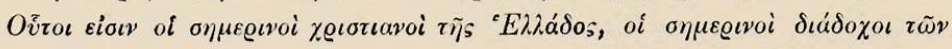

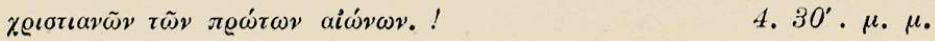




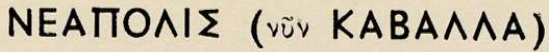

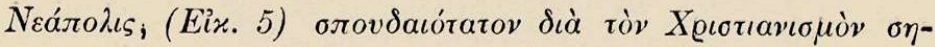

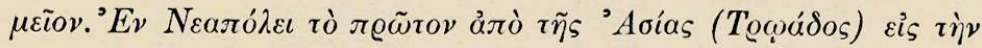

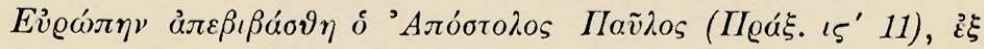

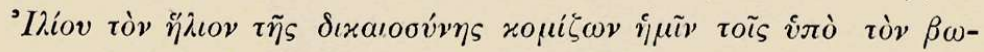

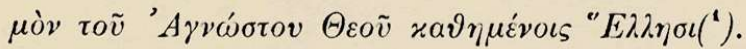

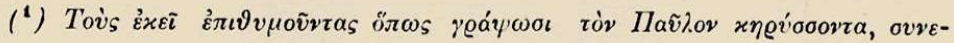

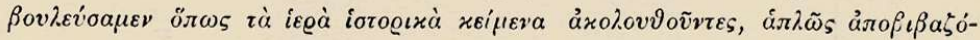

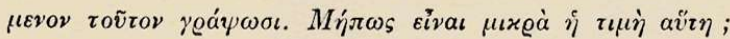

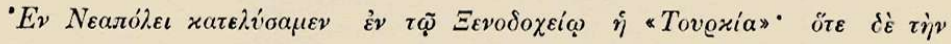

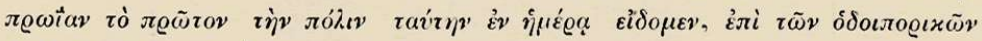

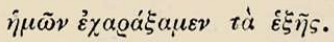

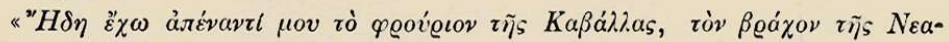

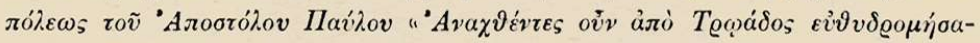

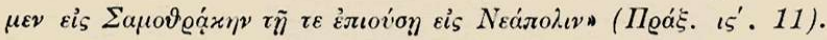

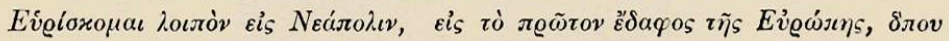

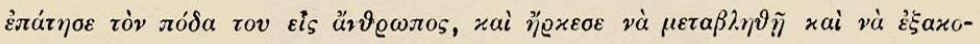

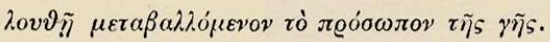

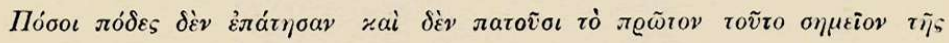

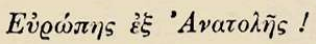

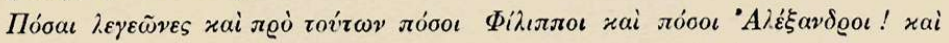

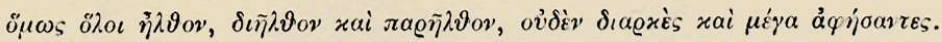

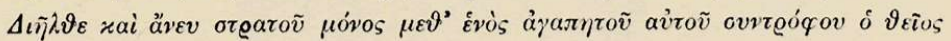

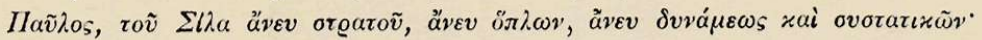

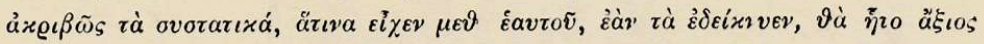

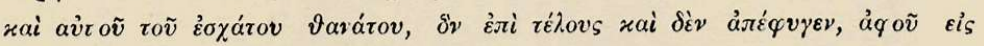

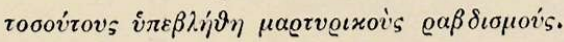

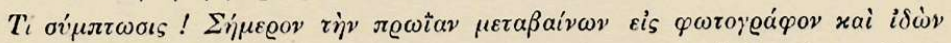

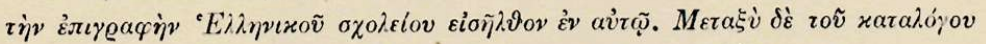

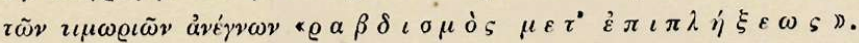

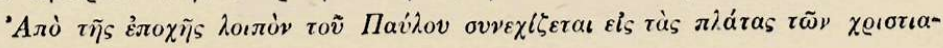
$\tau \tilde{\omega} \nu$ i $\alpha \dot{\imath} \tau \dot{\eta} \pi o \iota v \dot{\eta}$; 


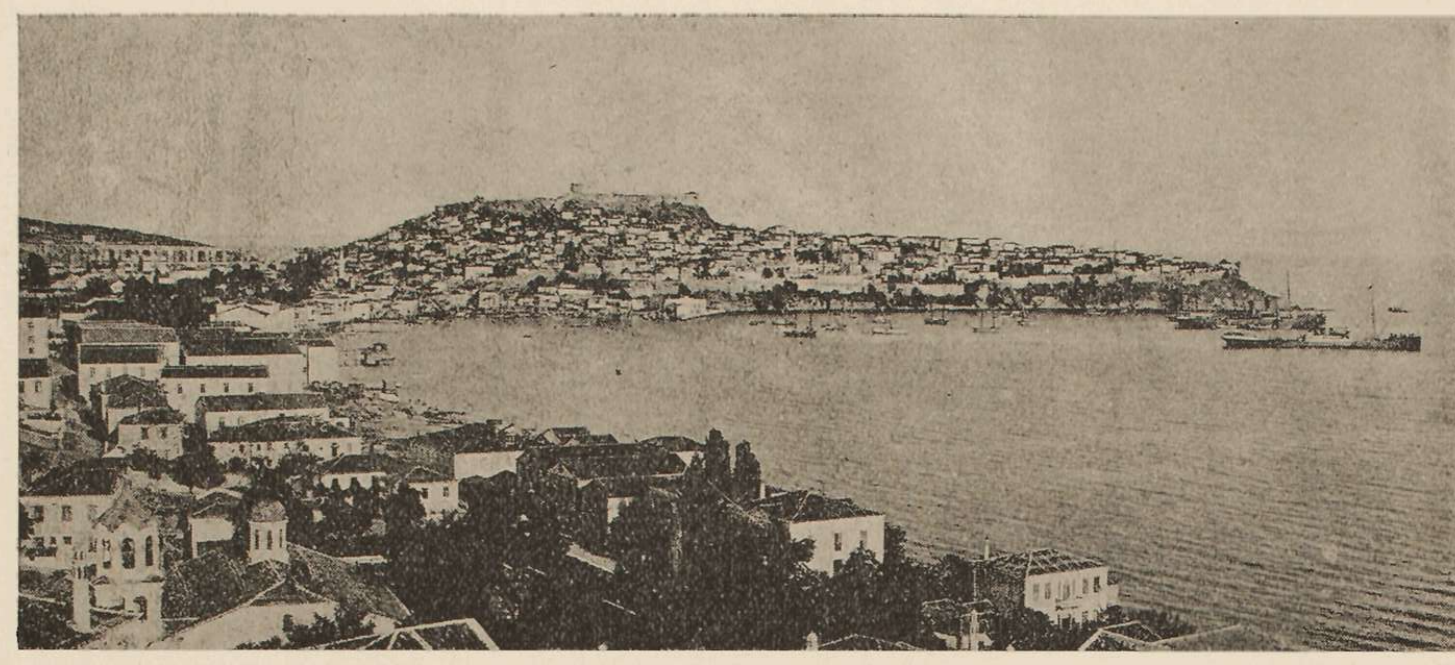

Eixìy 5.

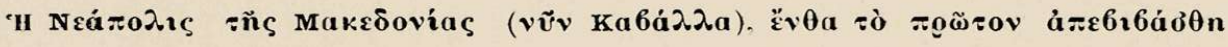

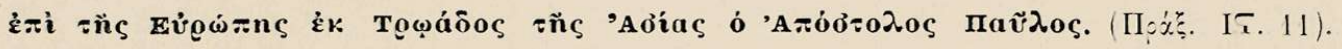




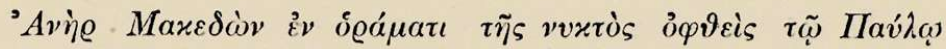

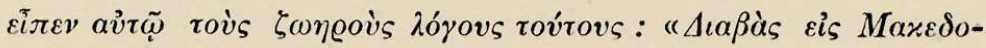

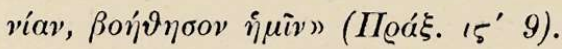

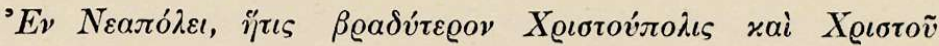

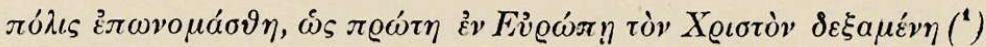

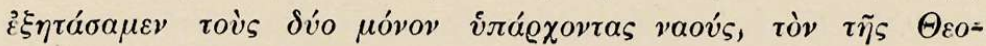

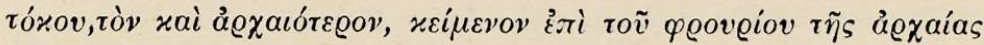

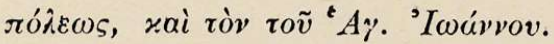

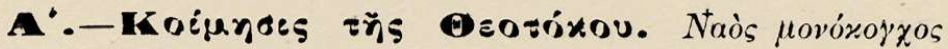

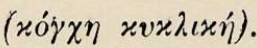

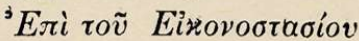

1G. $X C . \quad O$ BACINEYC TISN BACINEYONT $\Omega N$ KAI METAC APXIEPEYC. "ैQ vos $\dot{\varepsilon} \pi \iota \delta \iota \varrho \vartheta \omega \vartheta \dot{v} v$.

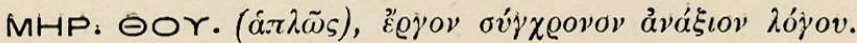

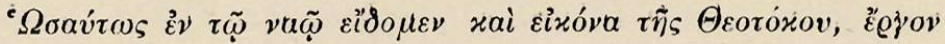

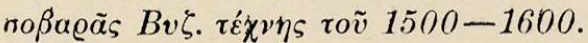

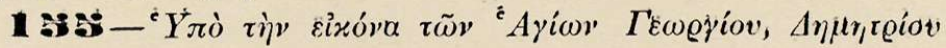

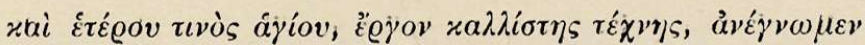

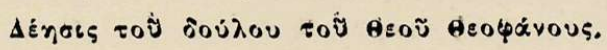

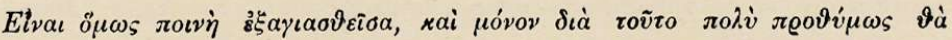

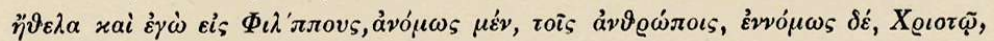
$\nu \grave{\alpha} \dot{\varepsilon} \varrho \alpha \beta \delta \iota \zeta o ́ \mu \eta \nu$.

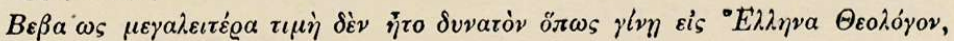

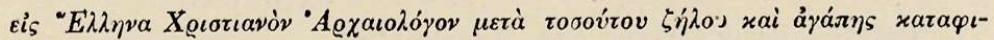

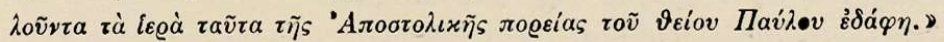

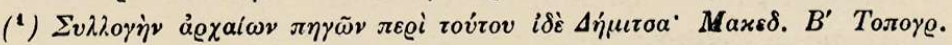

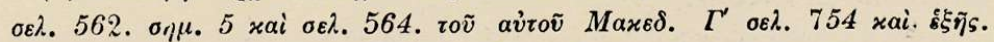

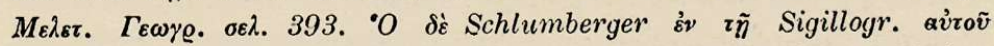

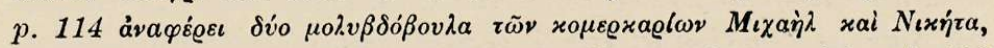

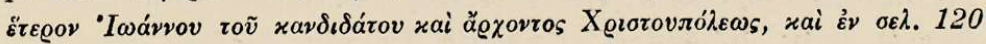

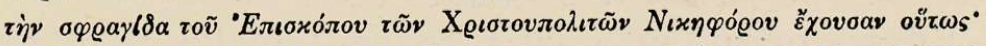

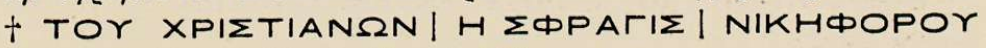

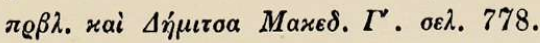




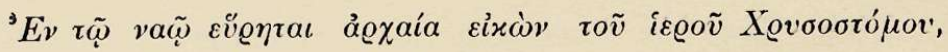

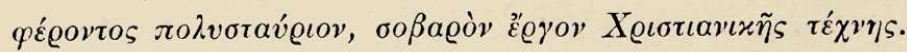

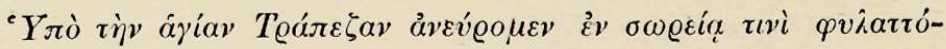

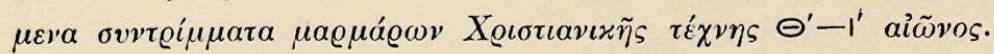

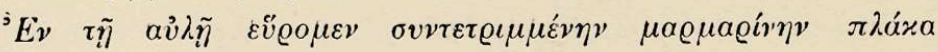

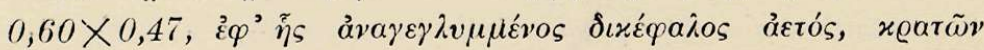

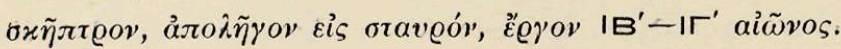

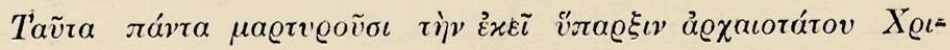

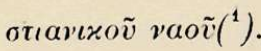

B'. - "A yeos 'I

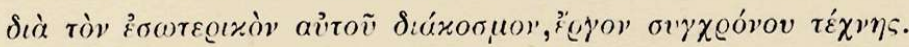

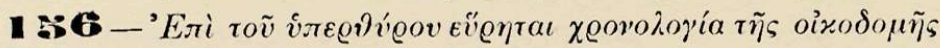
1857

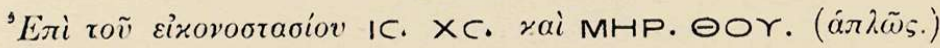

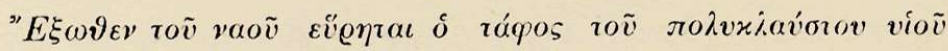

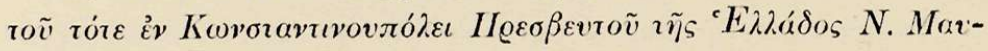

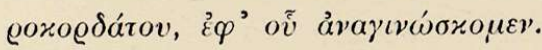

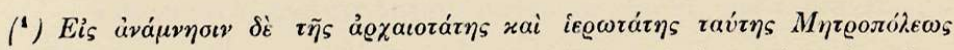

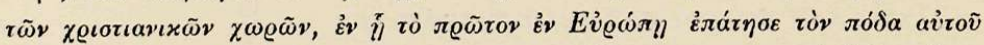

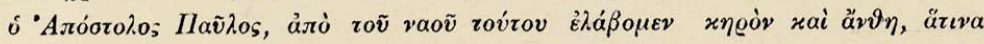

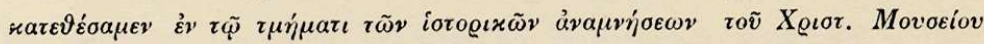
iл $\pi^{\circ} \varrho \iota \vartheta .3300$.

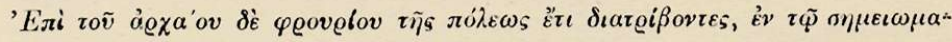

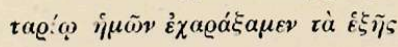

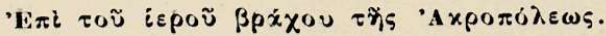

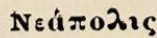

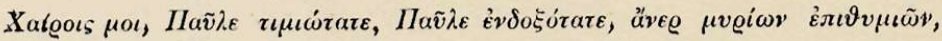

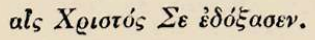

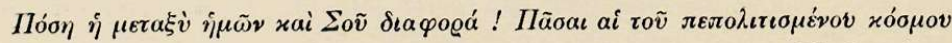

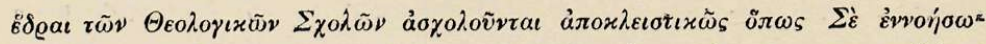

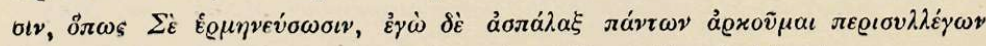

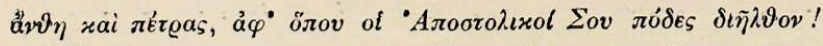

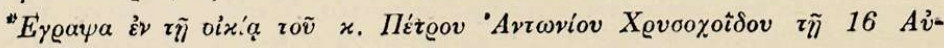
rov́arov 1902. 


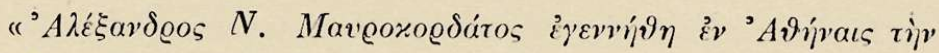

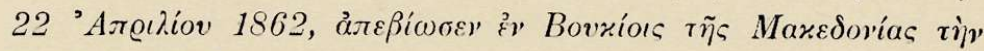
7 Maíov 1895).

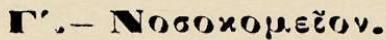

1 รัช - 'Eлi

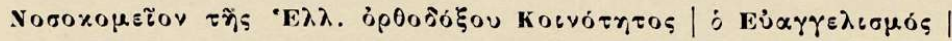

\section{0}

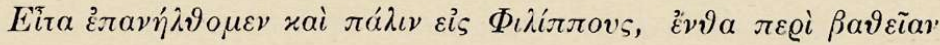

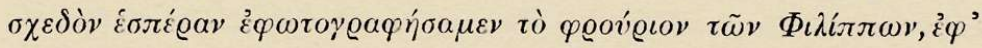

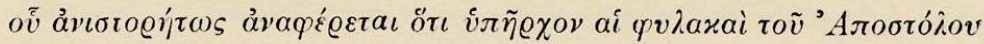

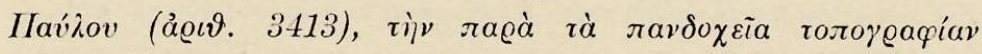

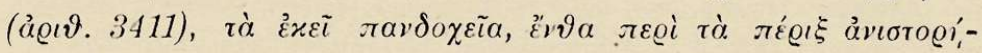

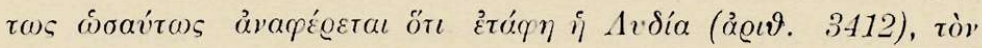

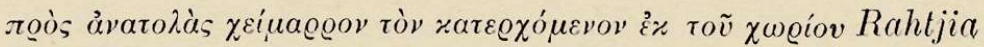

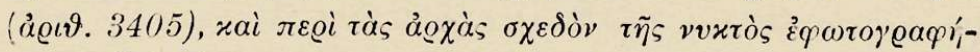

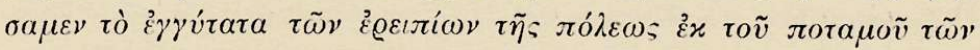

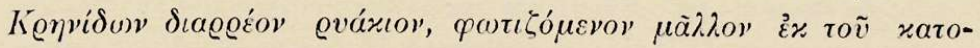

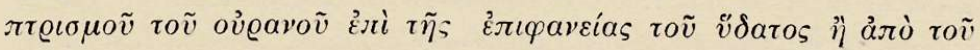

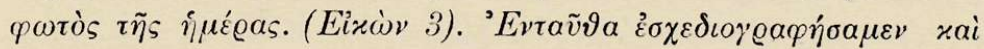

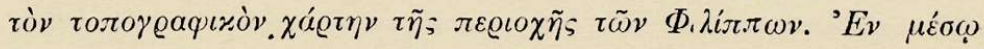

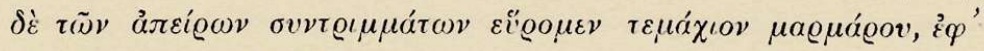

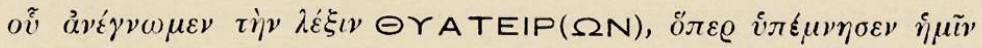

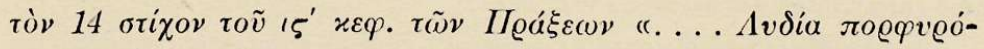

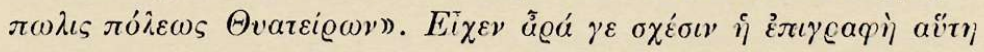

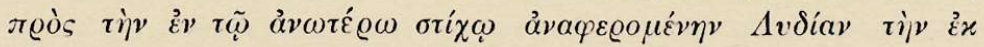

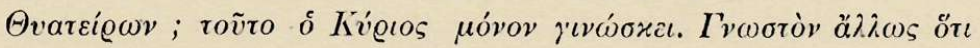

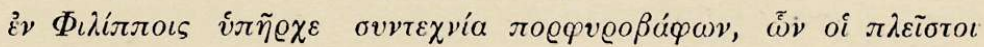

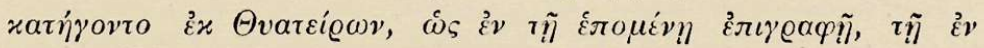

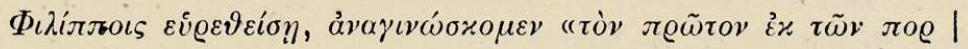

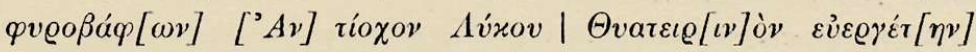

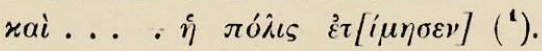

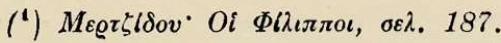




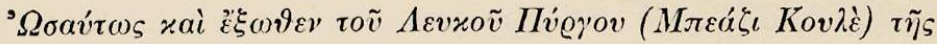

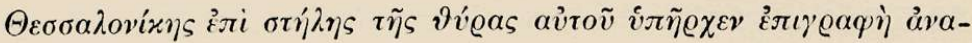

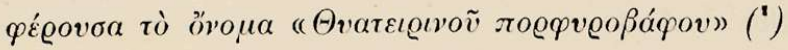

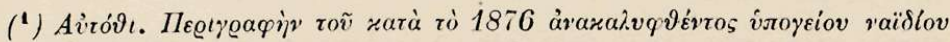

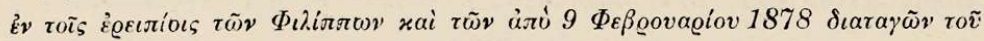

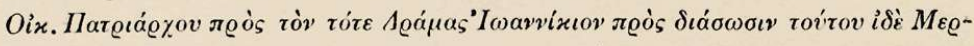

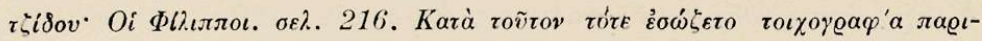

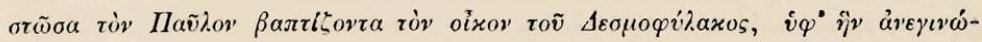

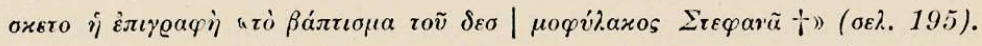

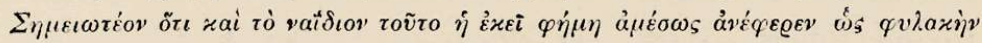

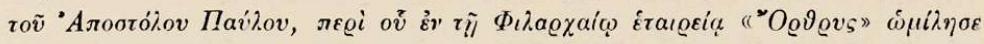

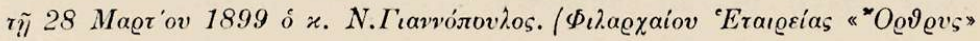

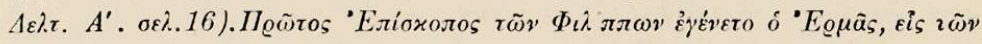

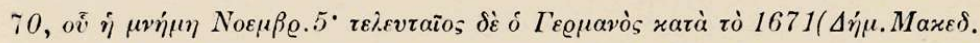

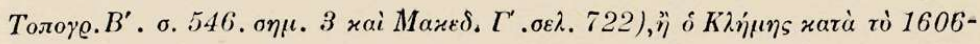

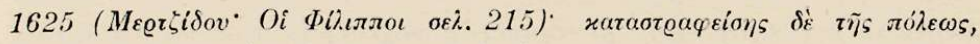

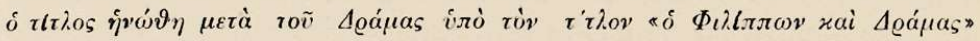

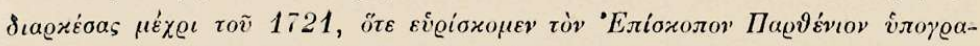

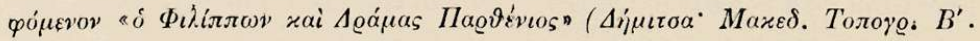

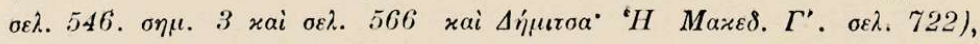

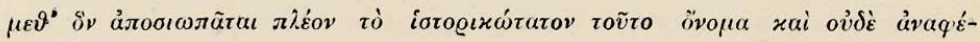

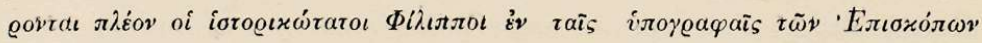

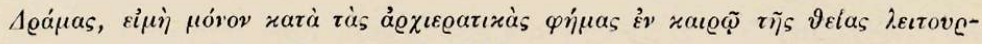

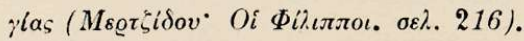

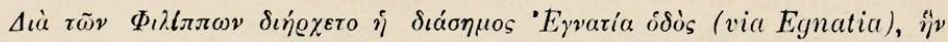

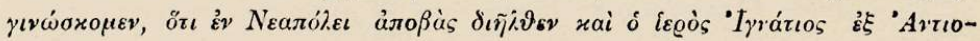

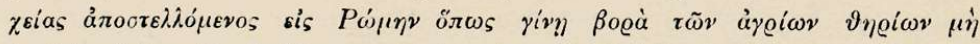

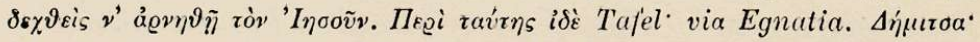

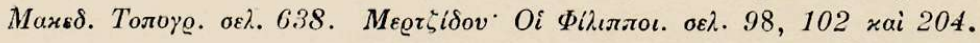

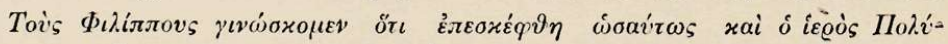

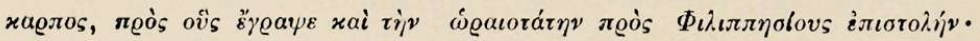

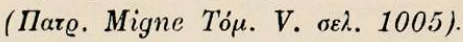

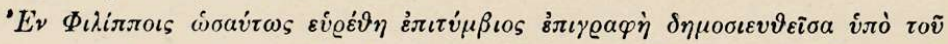

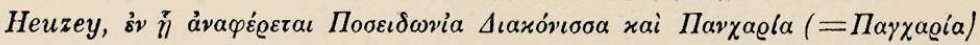

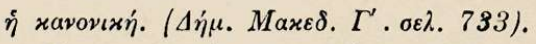

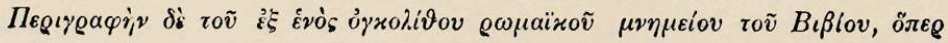




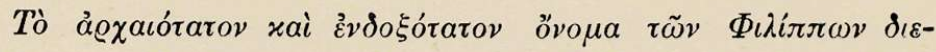

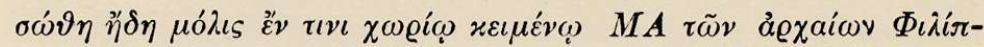

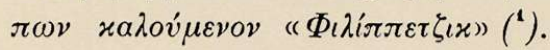

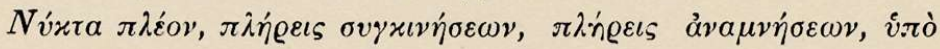

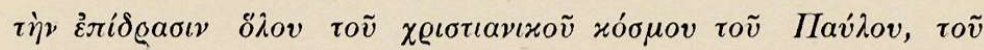

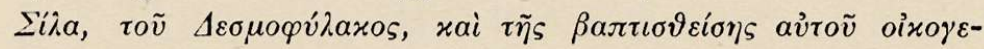

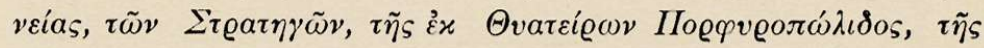

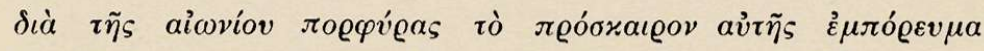

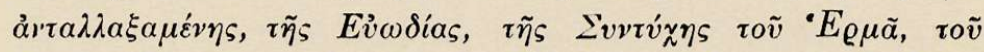

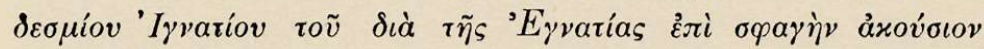

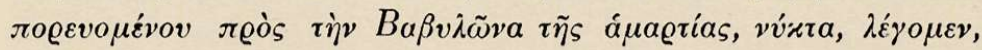

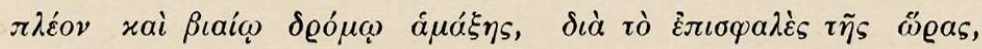

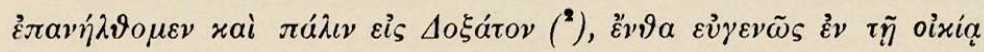

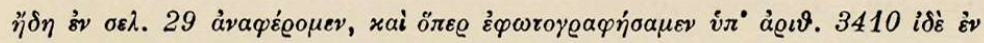

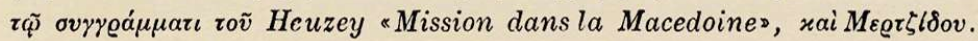

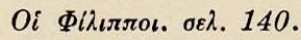

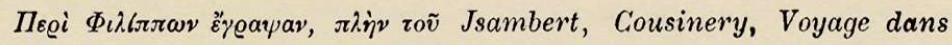
la Macedoine 1831, rov $W$. Leake, London 1835, тоv P. Bellon,

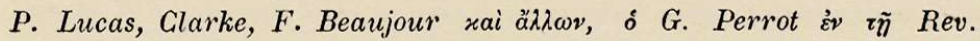

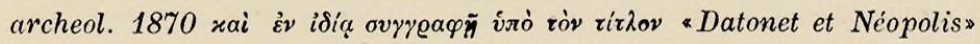

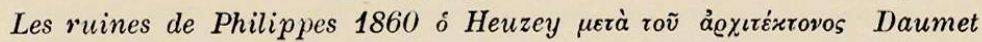

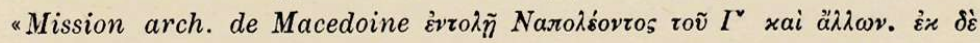

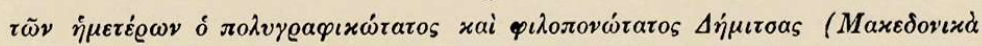

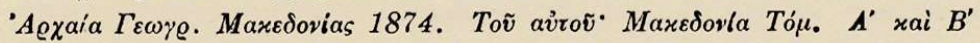

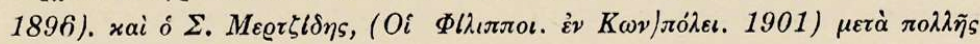

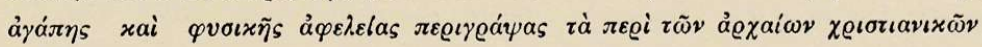

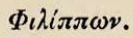

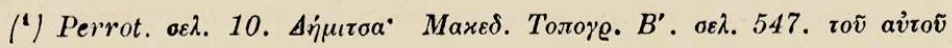

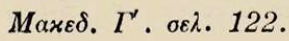

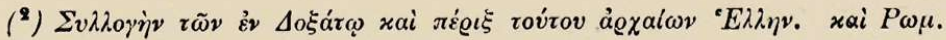

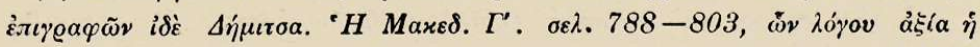

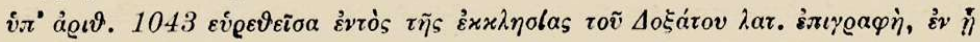

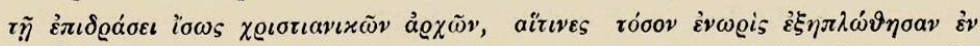

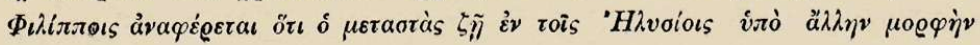




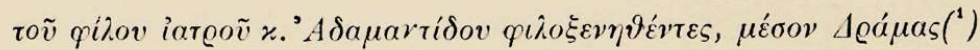

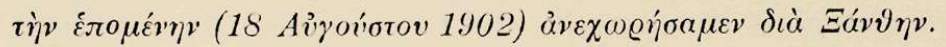

\section{$\equiv A N \odot H$}

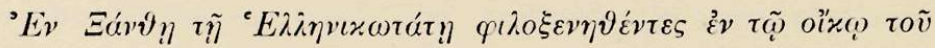

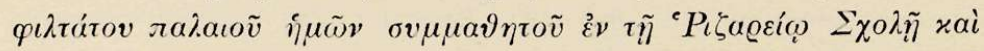

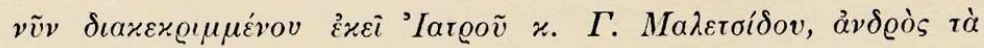

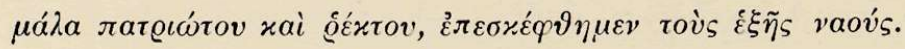

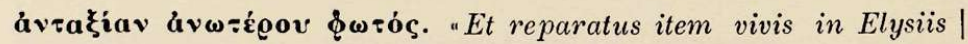
Sic plocitum est divis all]tera vivere forma | qui bene de Supero [l]umine sit meritus».

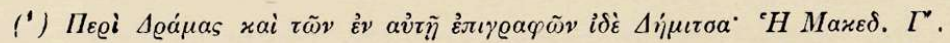

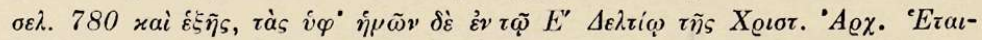

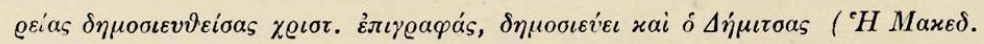

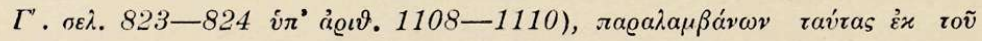

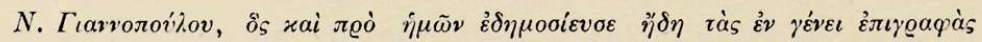

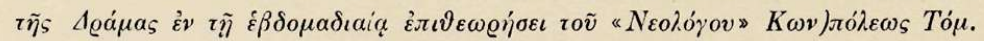

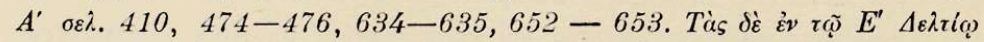

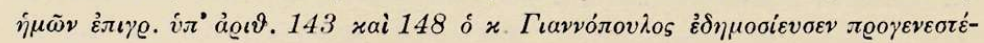

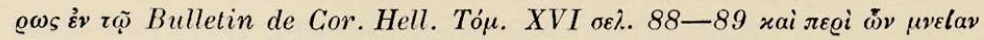

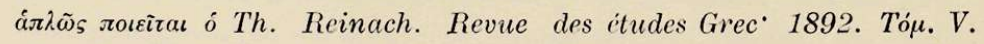

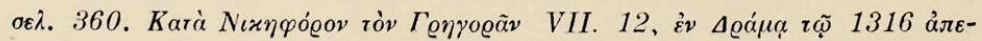

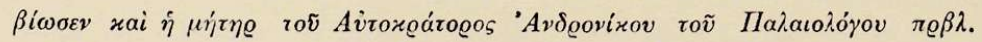

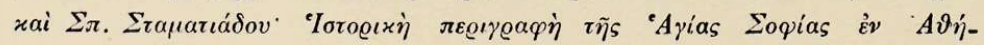
vaıs 1865 oв 31 . 31 .

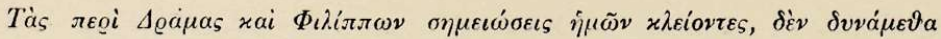

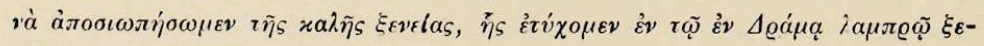

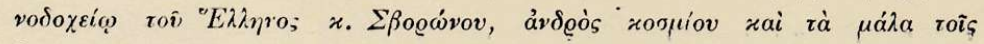

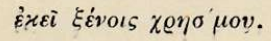




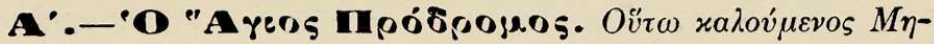

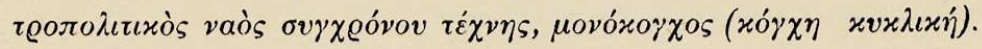

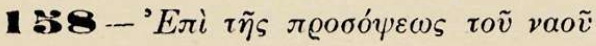

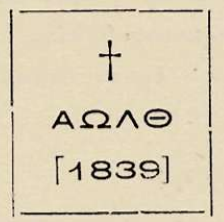

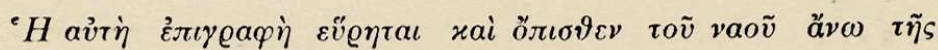

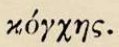

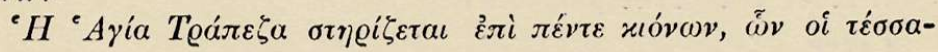

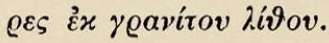

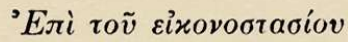

\section{IC. XC. O ПANTOKPAT $\Omega P$

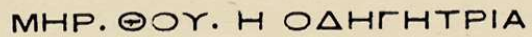

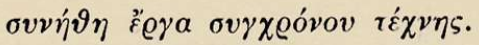

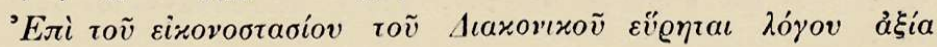

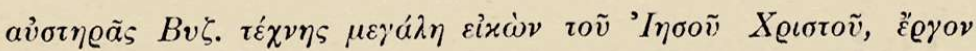

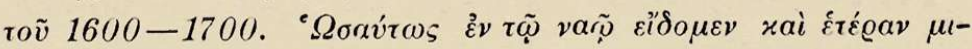

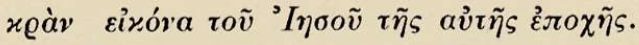

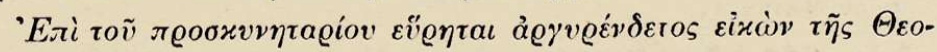

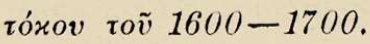

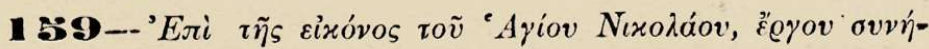

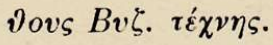

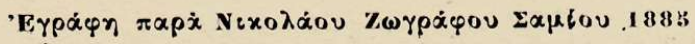

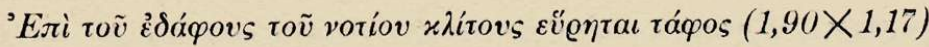

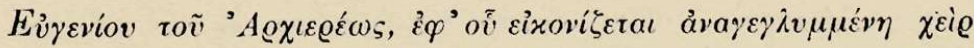

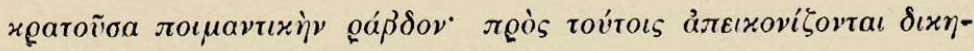




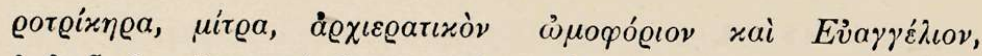

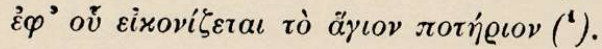

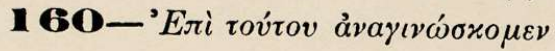

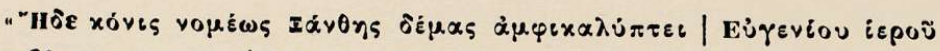

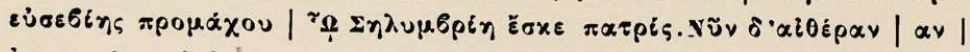

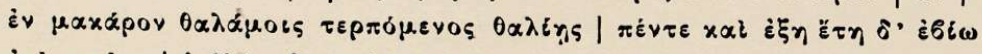

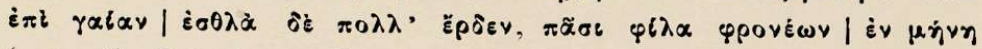

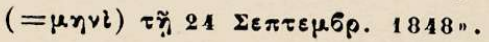

\section{B'. - 'O Tağeápxns.}

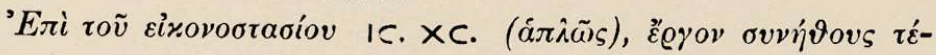

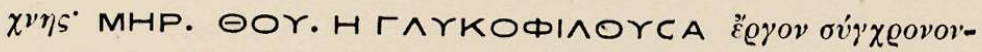

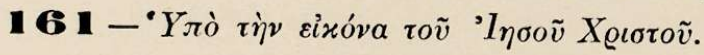

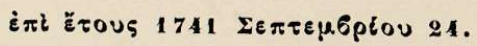

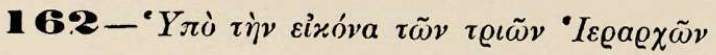

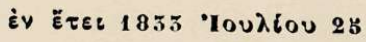

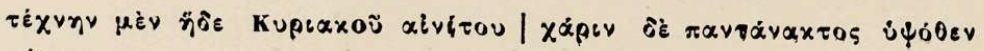

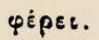

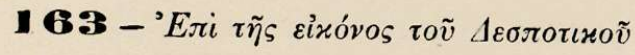

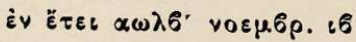

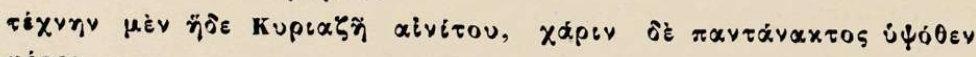

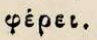

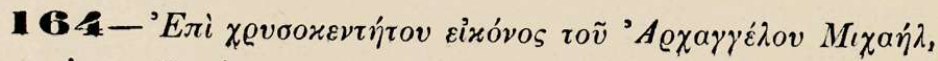

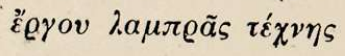

. $\omega \omega_{\lambda} \theta$ Matov 1.

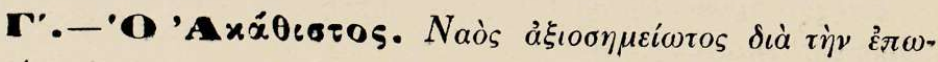
vvpíav,

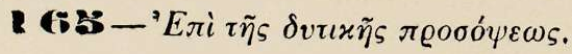

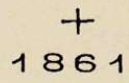

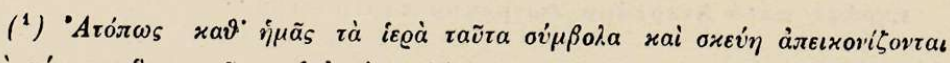

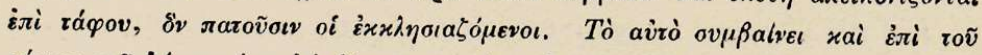

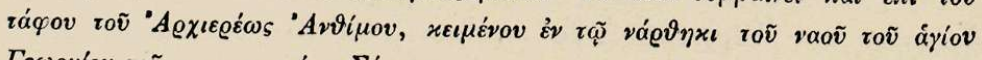

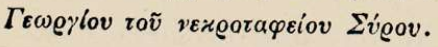


$166-E i s$ เò $v \pi \operatorname{r} \varrho \vartheta v \varrho o v$

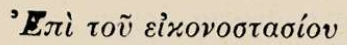

1860

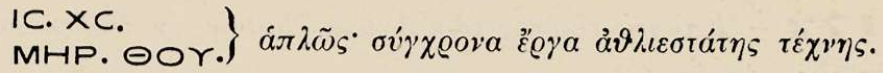

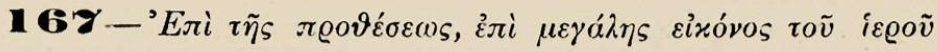

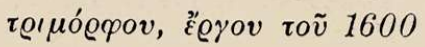

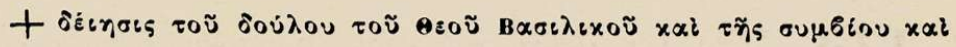

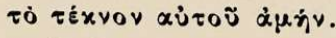

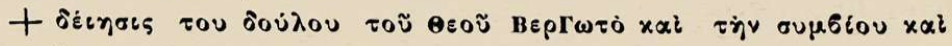

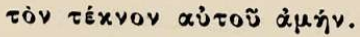

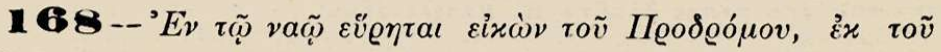

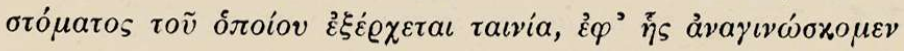

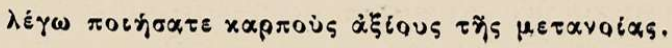

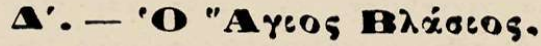

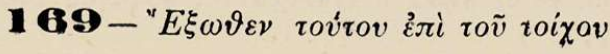

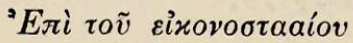

$$
+1828
$$

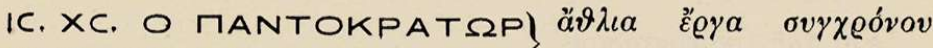

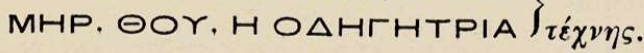

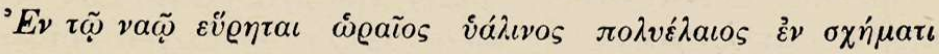

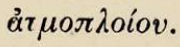

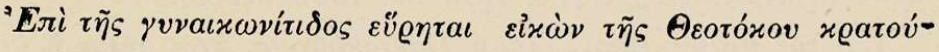

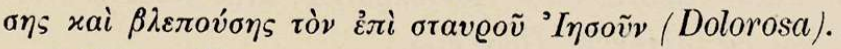

1 ชО - 'E Ei

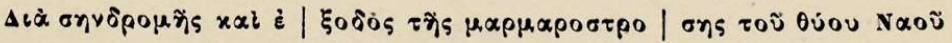

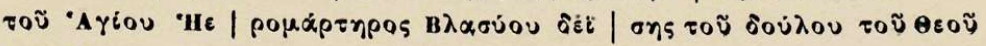
Mavó̀n.

\section{E'--.O "Aryeos Teẃoyeos.}

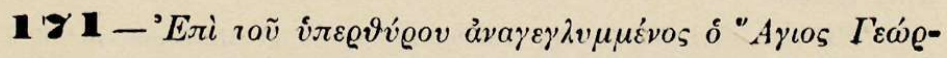

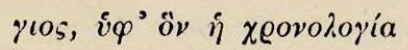




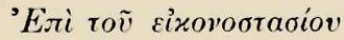

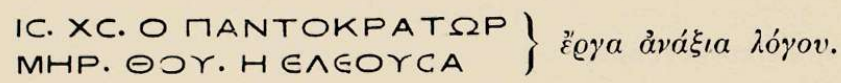

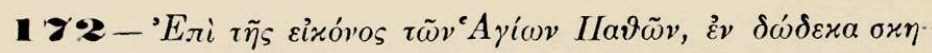

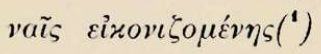

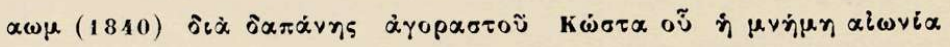

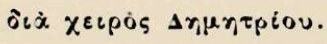

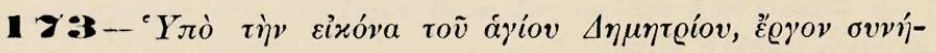
$\left.\vartheta \circ \vartheta_{\varsigma} B v \zeta . \tau \dot{\varepsilon} \chi\right\urcorner \eta \varsigma$

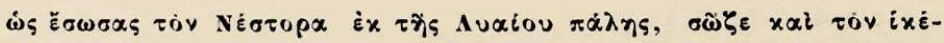

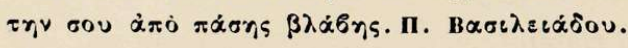

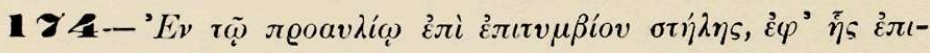

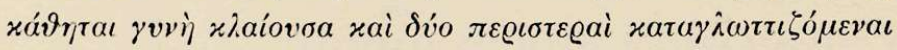

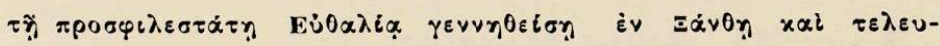

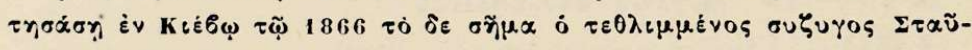

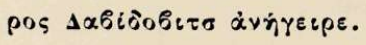

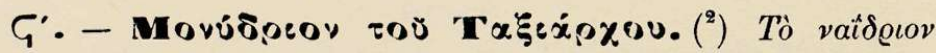

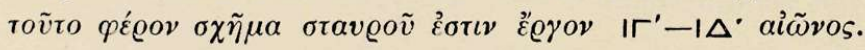

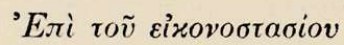

1C. $X$ C. OBACINEYC T $\Omega N$ BACINEYONT $\Omega N$ KAI

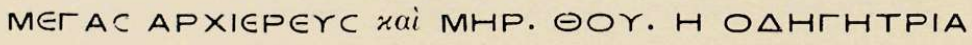

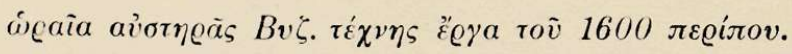

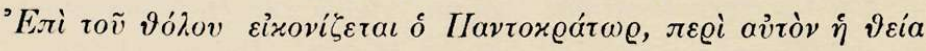

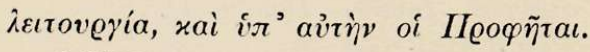

173 - Eлi

1836

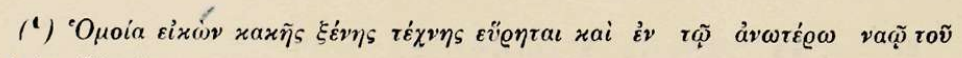
'A jíov Bhasiov.

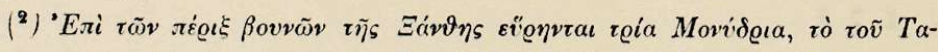

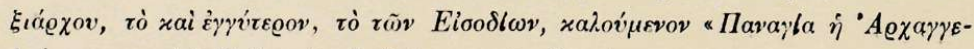

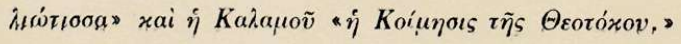




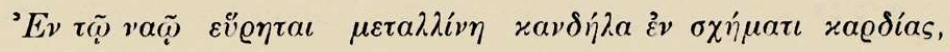

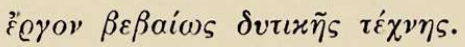

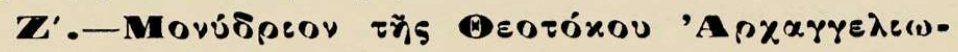

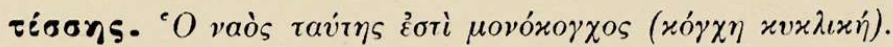

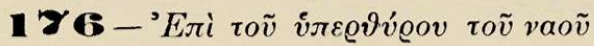

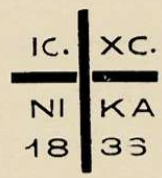

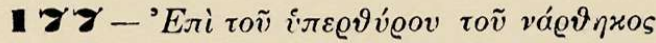

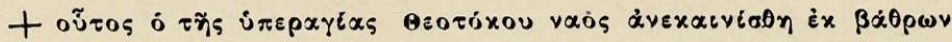

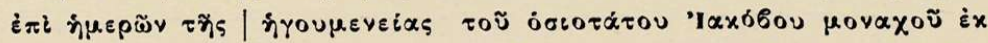

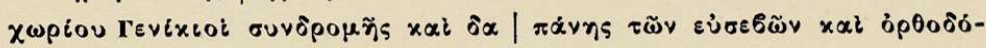

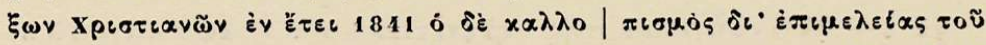

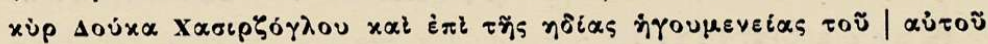

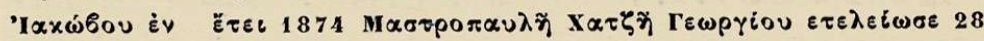
bx. $\mid 1874$.

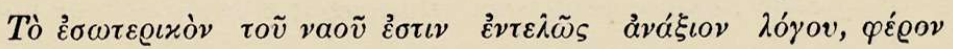

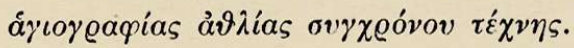

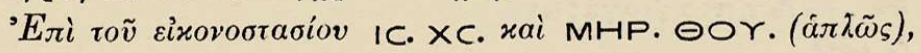

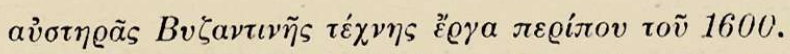

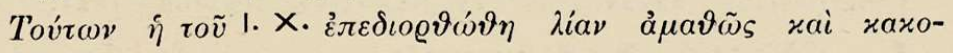
$\tau \dot{\chi} \chi \nu \omega s$.

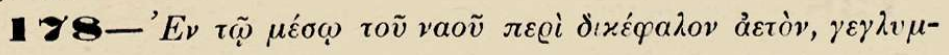

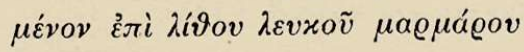

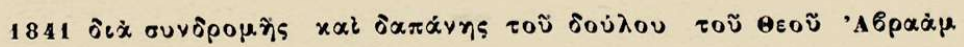

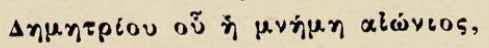

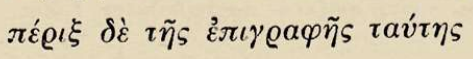

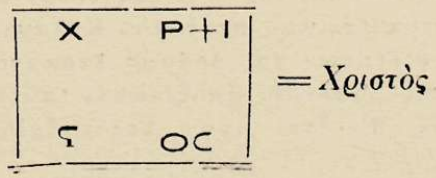




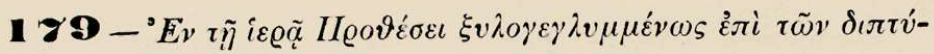
$\chi \omega v^{\cdot} \dot{\varepsilon} \pi i \tau \tilde{\eta} s \mu i \tilde{\alpha} s \tau v \chi \tilde{\eta} s$

$\triangle I A X P \quad N \in O \Phi(=\dot{o} ! \dot{\alpha} \chi \varepsilon ! \rho \dot{o} s \quad \mathrm{~N} \varepsilon 0 \hat{q})$

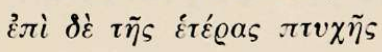

YTOY IEP(OMONAXOY) 'ZPKB' $(7122=1614)$

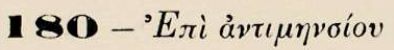

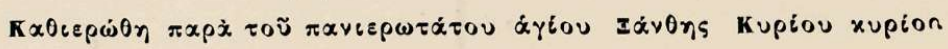

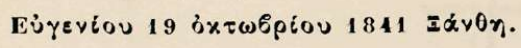

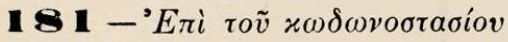

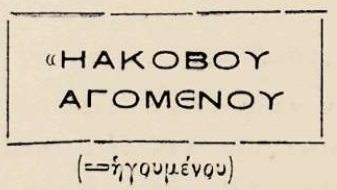

$\triangle O Y K A C$.

EחITPOTOC

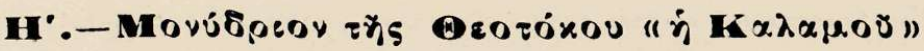

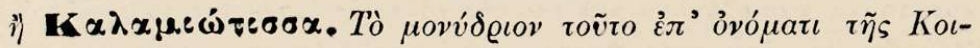

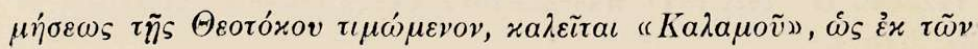

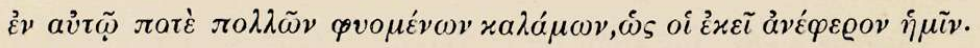

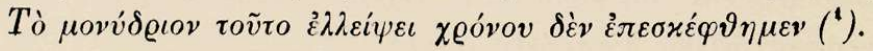

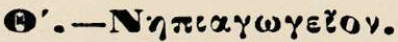

$182-' E \pi$ ' av̉นoṽ

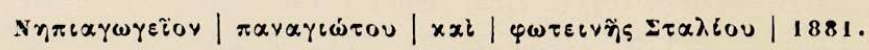

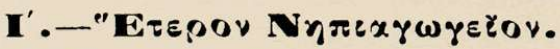

138 - 'E $E{ }^{\prime}$ av่นov

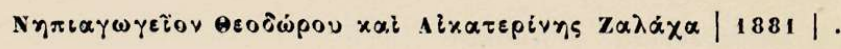

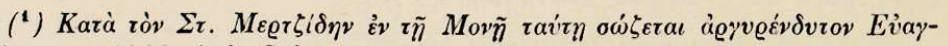

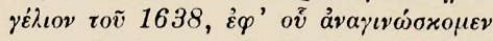

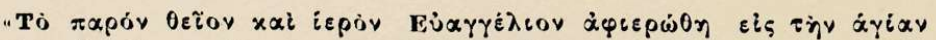

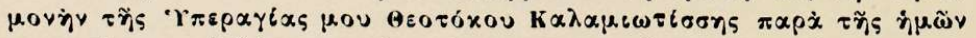

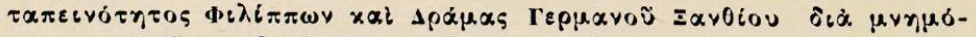

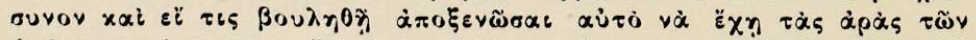

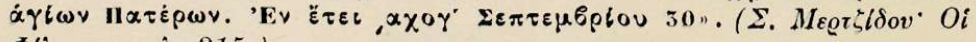
$\Phi\left(\lambda เ \pi \pi \circ \iota\right.$, $ө \lambda_{.}$215.) 


\section{A B $\triangle H P A$}

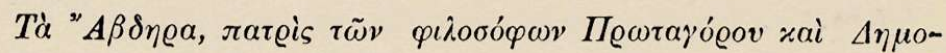

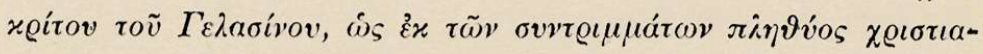

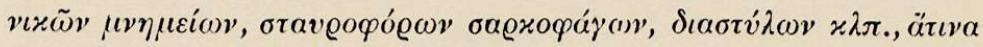

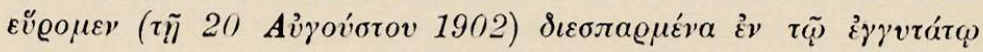

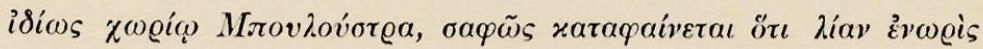

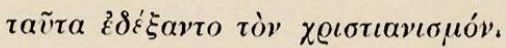

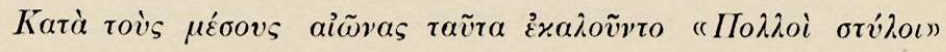

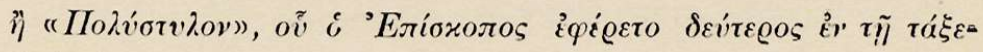

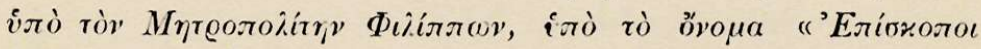
IIoһvбrúkov» ( $\left.{ }^{1}\right)$.

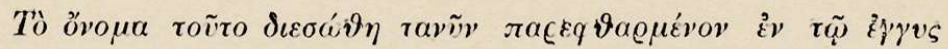

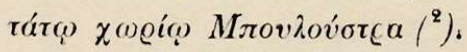

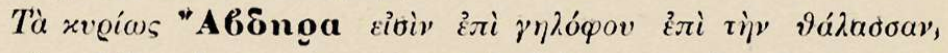

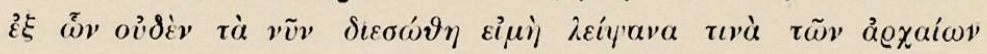

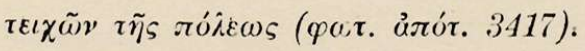

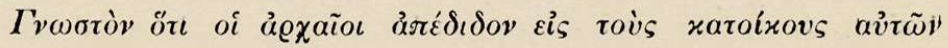

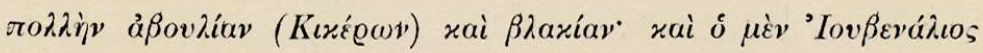

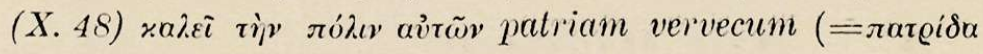

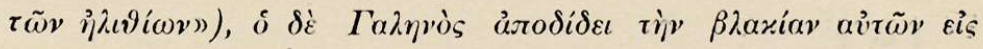

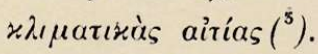

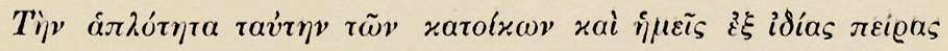

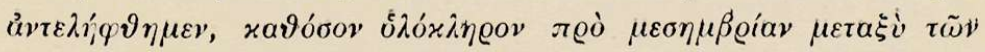
жатої

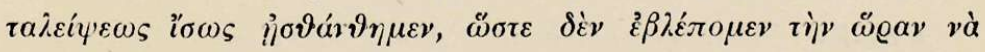

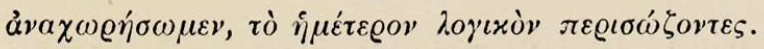

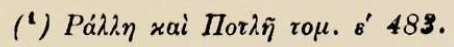

(2) $M \varepsilon \lambda \varepsilon \varepsilon i o v ~ \Gamma \varepsilon \omega \gamma \varrho \alpha \varphi . ~ \sigma \varepsilon \lambda .418$.

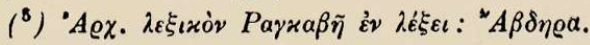




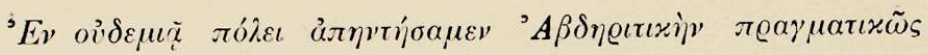

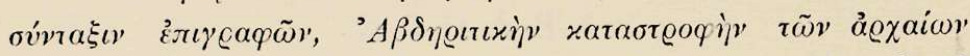

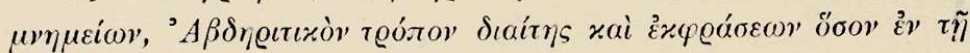

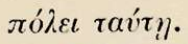

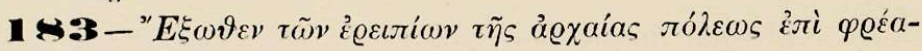

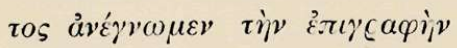

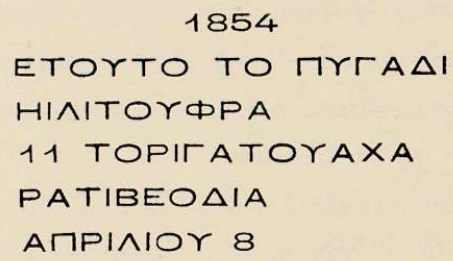

\section{M $\Pi$ O $\triangle O Y \Sigma T P A$}

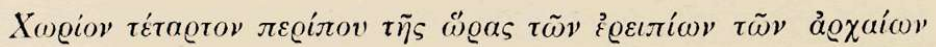
'A

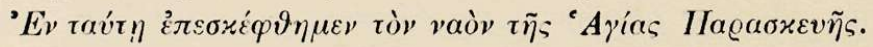

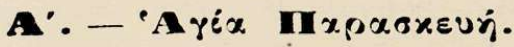

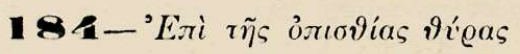

$$
+1845
$$

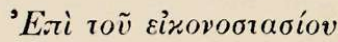

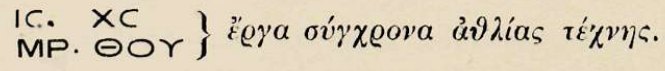

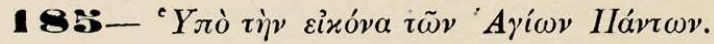

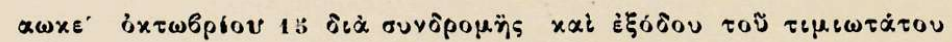

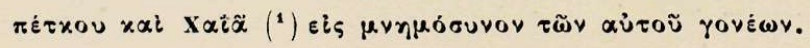

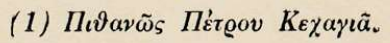




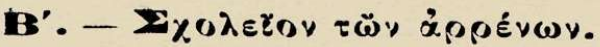

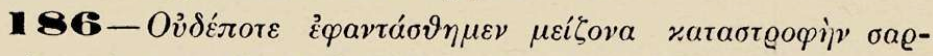

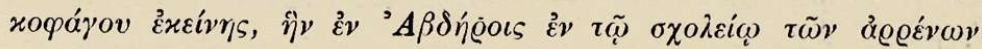

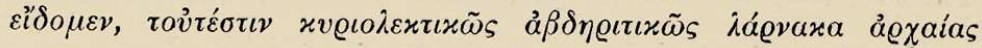

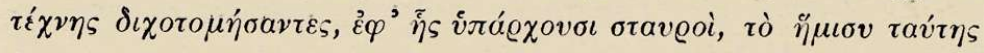

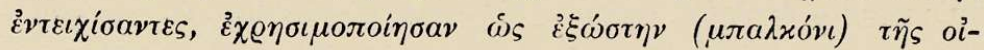

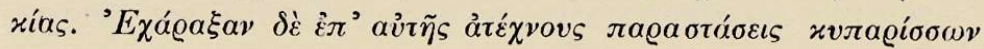

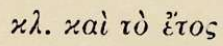

$$
\text { " } 1850 \text { " }
$$

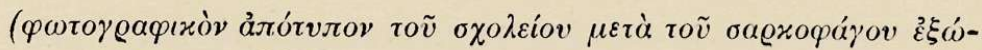

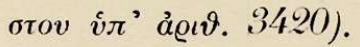

$18 \boldsymbol{8}$-'Eлi

I NIYON ANOMHMA| TA KE MH MO NO WIN

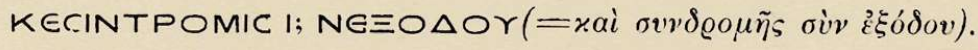
KOCTANTHNOY IEPE $\Omega C \in T \Omega C$. A WK

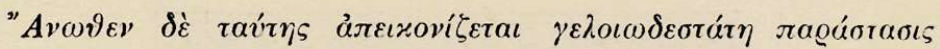

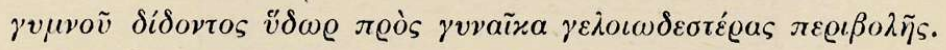

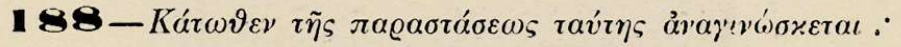

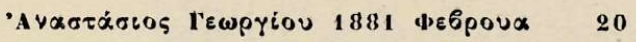

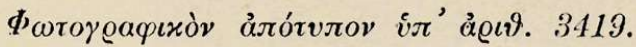

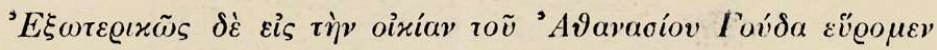

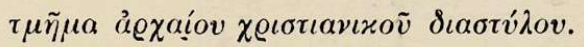

\section{ГIEN ITZE}

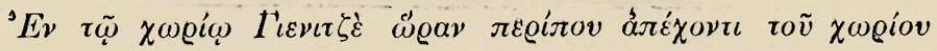

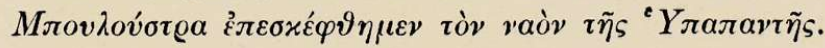

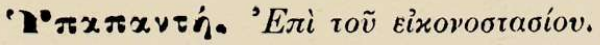

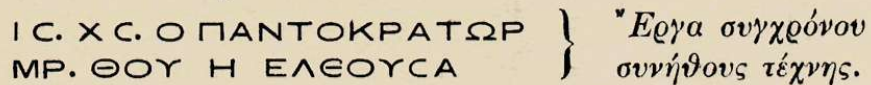




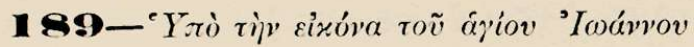

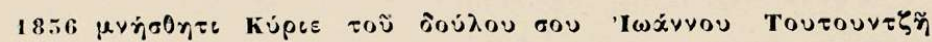

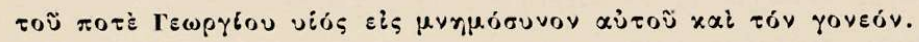

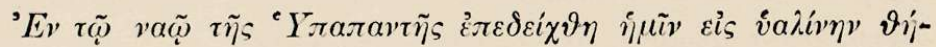

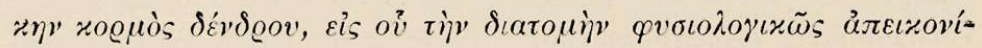

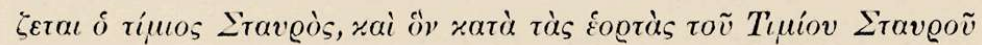

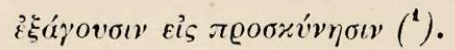

(1) "O

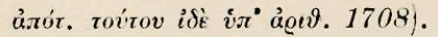

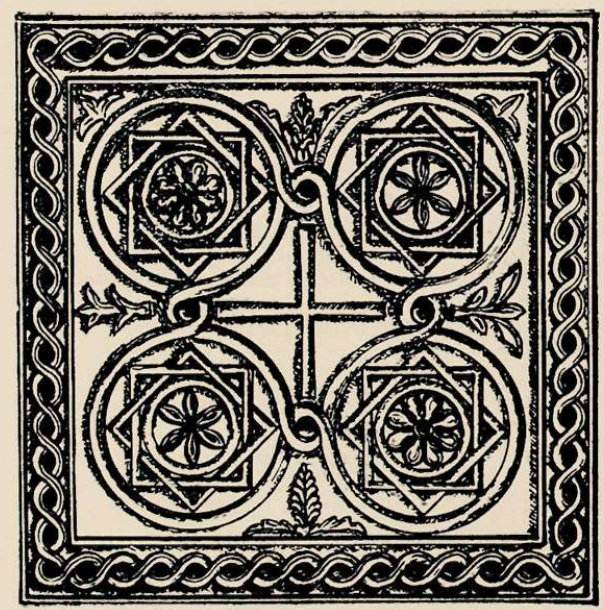

